\title{
The CoRoT satellite in flight: description and performance ${ }^{\star}$
}

\author{
M. Auvergne ${ }^{1}$, P. Bodin ${ }^{2}$, L. Boisnard ${ }^{2}$, J.-T. Buey ${ }^{1}$, S. Chaintreuil ${ }^{1}$, G. Epstein ${ }^{1}$, M. Jouret ${ }^{2}$, T. Lam-Trong ${ }^{2}$,
} P. Levacher ${ }^{3}$, A. Magnan ${ }^{3}$, R. Perez ${ }^{2}$, P. Plasson ${ }^{1}$, J. Plesseria ${ }^{11}$, G. Peter ${ }^{4}$, M. Steller ${ }^{10}$, D. Tiphène ${ }^{1}$, A. Baglin ${ }^{1}$, P. Agogué ${ }^{2}$, T. Appourchaux ${ }^{5}$, D. Barbet ${ }^{5}$, T. Beaufort ${ }^{9}$, R. Bellenger ${ }^{1}$, R. Berlin $^{4}$, P. Bernardi ${ }^{1}$, D. Blouin ${ }^{3}$, P. Boumier ${ }^{5}$, F. Bonneau ${ }^{2}$, R. Briet ${ }^{2}$, B. Butler ${ }^{9}$, R. Cautain ${ }^{3}$, F. Chiavassa ${ }^{2}$, V. Costes ${ }^{2}$, J. Cuvilho ${ }^{12}$, V. Cunha-Parro ${ }^{1}$, F. De Oliveira Fialho ${ }^{1}$, M. Decaudin ${ }^{5}$, J.-M. Defise ${ }^{11}$, S. Djalal ${ }^{2}$, A. Docclo ${ }^{1}$, R. Drummond ${ }^{13}$, O. Dupuis ${ }^{1}$, G. Exil ${ }^{1}$, C. Fauré ${ }^{2}$, A. Gaboriaud ${ }^{2}$, P. Gamet ${ }^{2}$, P. Gavalda ${ }^{2}$, E. Grolleau ${ }^{1}$, L. Gueguen ${ }^{1}$, V. Guivarc' 'h ${ }^{1}$, P. Guterman ${ }^{3}$, J. Hasiba ${ }^{10}$, G. Huntzinger ${ }^{1}$, H. Hustaix ${ }^{2}$, C. Imbert ${ }^{2}$, G. Jeanville ${ }^{1}$, B. Johlander ${ }^{9}$, L. Jorda ${ }^{3}$, P. Journoud ${ }^{1}$, F. Karioty ${ }^{1}$, L. Kerjean ${ }^{2}$, L. Lafond ${ }^{2}$, V. Lapeyrere ${ }^{1}$, P. Landiech ${ }^{2}$, T. Larqué ${ }^{2}$, P. Laudet ${ }^{2}$, J. Le Merrer ${ }^{3}$, L. Leporati ${ }^{3}$, B. Leruyet ${ }^{1}$, B. Levieuge ${ }^{1}$, A. Llebaria ${ }^{3}$, L. Martin ${ }^{3}$, E. Mazy ${ }^{11}$, J.-M. Mesnager ${ }^{2}$, J.-P. Michel ${ }^{1}$, J.-P. Moalic ${ }^{5}$, W. Monjoin ${ }^{1}$, D. Naudet ${ }^{1}$, S. Neukirchner ${ }^{10}$, K. Nguyen-Kim ${ }^{5}$, M. Ollivier ${ }^{5}$, J.-L. Orcesi ${ }^{5}$, H. Ottacher ${ }^{10}$, A. Oulali ${ }^{1}$, J. Parisot ${ }^{1}$, S. Perruchot ${ }^{3}$, A. Piacentino ${ }^{1}$, L. Pinheiro da Silva ${ }^{1}$, J. Platzer ${ }^{1}$, B. Pontet ${ }^{2}$, A. Pradines ${ }^{2}$, C. Quentin ${ }^{3}$, U. Rohbeck ${ }^{8}$, G. Rolland ${ }^{2}$, F. Rollenhagen ${ }^{4}$, R. Romagnan ${ }^{1}$, N. Russ ${ }^{4}$, R. Samadi ${ }^{1}$, R. Schmidt ${ }^{1}$, N. Schwartz ${ }^{1}$, I. Sebbag ${ }^{2}$, H. Smit ${ }^{9}$, W. Sunter ${ }^{9}$,

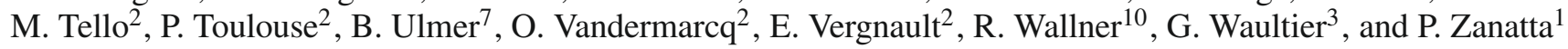

$$
\text { (Affiliations can be found after the references) }
$$

Received 26 August 2008 / Accepted 13 January 2009

\section{ABSTRACT}

Context. CoRoT is a space telescope dedicated to stellar seismology and the search for extrasolar planets. The mission is led by the CNES in association with French laboratories and has a large international participation. The European Space Agency (ESA), Austria, Belgium, and Germany contribute to the payload, and Spain and Brazil contribute to the ground segment. Development of the spacecraft, which is based on a PROTEUS low earth orbit (LEO) recurrent platform, commenced in October 2000, and the satellite was launched on December 27, 2006.

Aims. The instrument and platform characteristics prior to launch have been described in ESA publication (SP-1306). In the present paper we explain the behaviour in flight, based on raw and corrected data.

Methods. Five runs have been completed since January 2007. The data used here are essentially those acquired during the commissioning phase and from a long run that lasted 146 days. These enable us to give a complete overview of the instrument and platform behaviour for all environmental conditions. The ground based data processing is not described in detail because the most important method has been published elsewhere. Results. We show that the performance specifications are easily satisfied when the environmental conditions are favourable. Most of the perturbations, hence data corrections, are related to LEO perturbations: high energy particles inside the South Atlantic Anomaly (SAA), eclipses and temperature variations, and line of sight fluctuations due to the attitude control system. Straylight due to the reflected light from the earth, which is controlled by the telescope and baffle design, appears to be negligible.

Key words. instrumentation: photometers - stars: planetary systems - stars: oscillations

\section{Introduction}

Physical insight into stellar internal structure depends on our ability to compare and adjust models to reproduce the observations. However there are more unknown quantities than measured quantities so that many uncertainties remain, preventing accurate tests of the hypotheses contained in the modelling process. Asteroseismology, i.e. the detection of eigenmodes of oscillations, provides new observables (frequencies and amplitudes of eigenmodes) with very high accuracy and increases the number of constraints on models. It has already proven to be

\footnotetext{
* The CoRoT space mission, launched on December 27th 2006, has been developed and is operated by CNES, with contributions from Austria, Belgium, Brazil, ESA, Germany and Spain. Four French laboratories associated with the CNRS (LESIA, LAM, IAS, OMP) collaborate with CNES on the satellite development. The authors are grateful to Ian Roxburgh for a careful reading of the manuscript.
}

a powerful tool to probe the interior of the Sun. Observations of other stars are needed to test different physical conditions and distinguish physical processes. Seismology measurements are performed in the bandwidth 0.1 to $10 \mathrm{mHz}$, covering both the pressure and gravity modes of stars. For stochastically excited modes, the frequency measurement precision depends on the length of the observation window, on the mode lifetime, on the amplitude, and on the $S / N$ in the Fourier spectrum. We expect mode lifetimes of about 5 days and amplitudes greater than $2 \mathrm{ppm}$; therefore, the target stars are observed for 150 days with a minimum $S / N$ of 15 (in terms of power spectral density) to yield a precision on frequency measurements of $0.1 \mu \mathrm{Hz}$. The $S / N$ should be reached in the frequency interval if the noise level remains of the order of $0.6 \mathrm{ppm}$ in the Fourier spectrum in 5 days of observation for a $5.7 \mathrm{~V}$ magnitude star.

With high-precision stellar photometry we can detect extrasolar planets by the transit method, measuring the decrease in 
the stellar flux when the star, the planet and the instrument are almost aligned. The flux decrease is given by

$\frac{\Delta F}{F} \propto\left(\frac{R_{\mathrm{p}}}{R_{\mathrm{s}}}\right)^{2}$

where $R_{\mathrm{p}}$ is the radius of the planet and $R_{\mathrm{S}}$ the radius of the parent star. If the impact parameter is equal to 0 , the transit duration is

$\operatorname{tr}=\frac{P}{\pi}\left(\frac{R_{\mathrm{s}}}{a}\right)$

where $P$ is the orbital period of the planet and $a$ the radius of the orbit supposed to be almost circular.

In addition to hundreds of Uranus-like or Jupiter-like planets, several telluric planets should be detected, if our hypotheses about accretion models and planets occurrence are correct. To reach this goal the required photometric precision is $7 . \times 10^{-4}$ for a $V=15.5 \mathrm{mag}$ star for one hour integration. Up to 12000 target stars, in a field of view of 4 square degrees, are simultaneously observed during a 150-day period. With at least five different fields of view, more than 60000 stars will be followed during 150-day over the whole mission. A short run, typically of 25 days, is performed between each 150-day run.

The layout of our paper is as follow. In Sect. 2 we review the mission status and the spacecraft characteristics. Then the environmental conditions for a LEO and the impact on the photometric data are recalled in Sect. 3. In addition some results obtained during the commissioning phase are presented. Section 4 briefly describes the payload architecture and gives details on the payload and satellite behaviour. We develop important features of some functional subsystems such as the attitude control system, the temperature control system and the detection chain. On board and ground based data processing are summarised in Sects. 5 and 6 and photometric performances are presented in Sect. 7 for the AsteroSeismology (AS) and Planet Finder (PF) channels. In Sect. 8 a brief summary closes the paper.

\section{The satellite}

The satellite was launched successfully on 27/12/2006 into a polar orbit. This orbit is so close to the target one that no orbit corrections were necessary. The inclination of the orbit plane, which is 90.002 degrees (for a target inclination of 89.184 degrees), produces a drift of the orbit plane of one degree per year toward increasing right ascension (RA). The apogee and perigee are respectively 911 and $888 \mathrm{~km}$. The orbital period is $6184 \mathrm{~s}$. Up to April 2008 five observing runs were successfully completed. Their durations are 59, 28, 157, 146, and 20 days, for respectively the IRa01, SRc01, LRc01, LRa01 and SRa01 (LR stands for long run, SR for short run, IR for initial run and the small letters a and c for galactic anti-centre or galactic-centre direction). For a complete overview of the mission see for example the CoRoT Book (Fridlund et al. 2006) and Boisnard \& Auvergne (2004).

The spacecraft is based on a generic PROTEUS platform (Landiech \& Douillet 2004) developed under a close collaboration between the Centre National d'Études Spatiales (CNES) and Alcatel Space Industry (now re-named Thales-Alenia Space). It consists not only of a generic platform but also of a generic ground segment. The platform was designed for LEO missions with a maximum altitude of $1300 \mathrm{~km}$. Its lifetime is 3 years, and elements sensitive to aging or radiation are sized to 5 years. The satellite is operated from the CoRoT Control Centre located

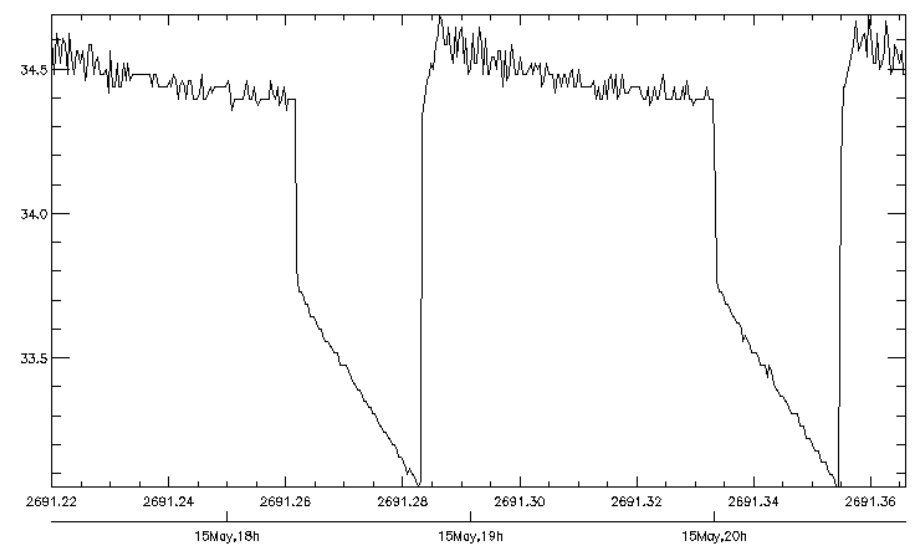

Fig. 1. Power supply voltage variations at eclipse ingress/egress on two orbits. Ordinate unit: Volts. Abscissa unit: Julian days. For all plots the Julian origin is 2000 January 1 , JD0 $=2451545$.

in Toulouse, sharing the facilities of the PROTEUS satellite family. The preparation of the observing runs, the payload command control, and the pre-processing of the scientific data are achieved by the CoRoT Mission Centre in collaboration with participating laboratories. A network of CNES ground stations (Kiruna, Aussaguel, Hartebeesthoek, Kourou) and one mission-specific secondary ground station located in Alcantara (Brazil) are used for communication with the satellite or reception of technical and scientific telemetry.

The interfaces for a dedicated mission are at satellite level between the platform and the payload and at ground level between the Control Centre and the specific Mission Centre. In the configuration adopted for CoRoT, several subsystems have been upgraded: Li-Ion battery, high capacity magneto torquer bars, and new star trackers (SED-16). The total weight is $626 \mathrm{~kg}$, including $300 \mathrm{~kg}$ for the payload. A mass memory provides a storage capacity of 2 Gbits at end of life for the payload and housekeeping data. The power is provided by two solar arrays that feed directly into the battery giving a non regulated voltage in the interval $23-37 \mathrm{~V}$. and a power of $1 \mathrm{~kW}$. The battery management is driven by autonomous software. An important functional chain is the attitude control system (ACS). At platform level the pointing is provided by star trackers, inertial wheels, magneto-torquers, and gyrometers, giving an angular stability of 16 arcsec. Since CoRoT's requirement is about 0.5 arcsec rms, to reach this value it was necessary to include ecartometry computations in the control loop based on the position of two stars on the payload focal plane in the control loop ecartometry.

\section{Environmental conditions}

As the orbit is at an altitude close to $900 \mathrm{~km}$, the Earth has an influence on the satellite and introduces perturbations on three characteristic time scales: the orbit (and harmonics), the day (and its first harmonic), and the seasons. The main perturbations are

- Eclipses. The transition light/penumbra/shadow and the reverse produces temperature variations, vibrations in the solar panels at the transition time, and voltage fluctuations when the power supply switches from the solar arrays to the battery (see Fig. 1).

- Gravity field and Earth's magnetic field. These induce perturbation torques on the satellite perturbing the satellite attitude.

- South atlantic anomaly (SAA). The magnetic field is not a perfect dipole, and the radiation belts are distorted above 


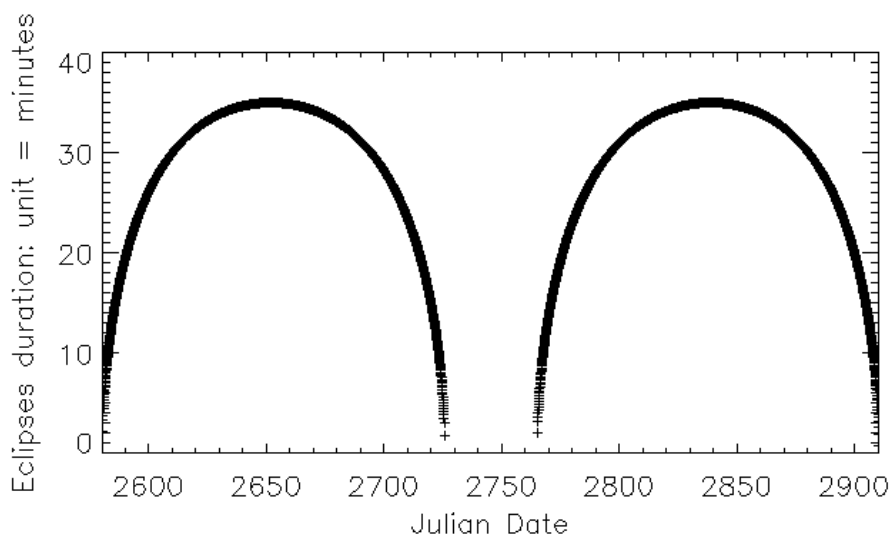

Fig. 2. Eclipse durations during the year 2007.

South America. This is because the centre of Earth's magnetic field offset by $450 \mathrm{~km}$ from the geographic centre. Trapped particles are essentially protons with energies in the interval $10 \mathrm{keV}$ to $300 \mathrm{MeV}$. The electron belts around the pole do not perturb the detectors thanks to a $10 \mathrm{~mm}$ aluminium shielding.

- Sun and Earth infrared emissivity. The Sun's position produces seasonal temperature variations, mainly through the focal box radiator. The Earth's infrared emission produces temperature variations on time scales of orbits and days.

- Earth albedo. The sunlight is reflected (or not) by the Earth depending on the region overflown, day/night region, cloudy zone, and ocean.

- Objects in LEO. At altitudes between 400 and $1500 \mathrm{~km}$, there are several thousands of satellites and debris larger than $10 \mathrm{~cm}$. When such an object is in the CoRoT FoV it can produce a local or global perturbation everywhere in the FoV.

\subsection{Eclipses}

The eclipse durations vary along a run. The eclipses are longer at the beginning or at the end of a run (just after or before the equinoxes). Near the solstices there are no eclipses for more than a month (see Fig. 2). The longest eclipse duration is $34.72 \mathrm{mn}$. All other effects are explained in the following.

\section{2. $S A A$}

The radiation flux, intensity of impact, and the number of pixels perturbed were studied during the commissioning period on images specifically acquired for this task. The detection method of impact in the image and results are given in Pinheiro da Silva et al. (2008). We recall the main results in the following. Comparisons with theoretical models show that protons are the dominant contribution, and the measured flux is in good agreement with the predicted one (see Fig. 3). The average imparted energy per impact (not to be confused with the proton energy) is $5160 \mathrm{e}^{-}$, and the most violent event generates more than $1.1 \mathrm{Me}^{-}$. The mean number of pixels disturbed in an impact is 7.9. It is also found that low energy events are dominated by secondary particles generated by protons in the surrounding material of the detectors. We define the limits of the SAA such that the probability of an impact inside a stellar image is less than $50 \%$. This corresponds to a proton flux of $200 \mathrm{p}^{+} / \mathrm{cm}^{2} / \mathrm{s}$ on the AS channel and $50 \mathrm{p}^{+} / \mathrm{cm}^{2} / \mathrm{s}$ on the PF channel. With this definition the percentage of time spent by the satellite inside the SAA is $7 \%$. It is a significant contribution to the duty cycle

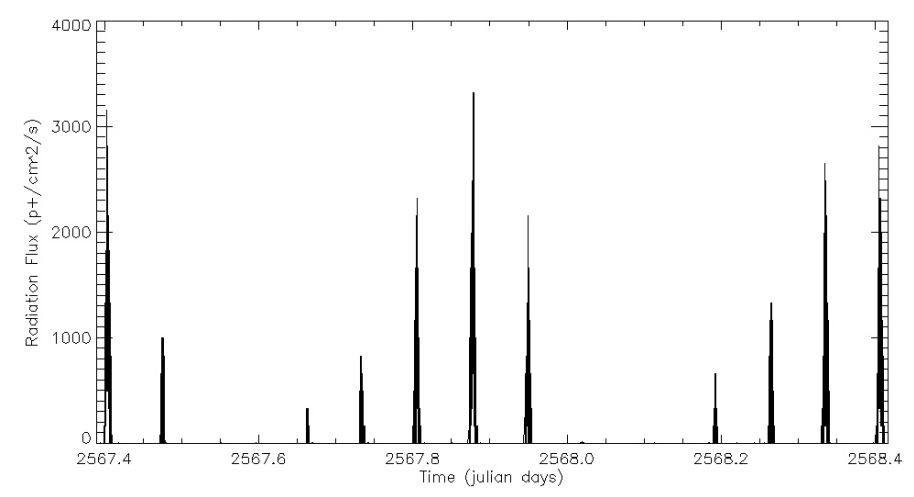

Fig. 3. Temporal evolution of the proton flux at the CoRoT altitude.

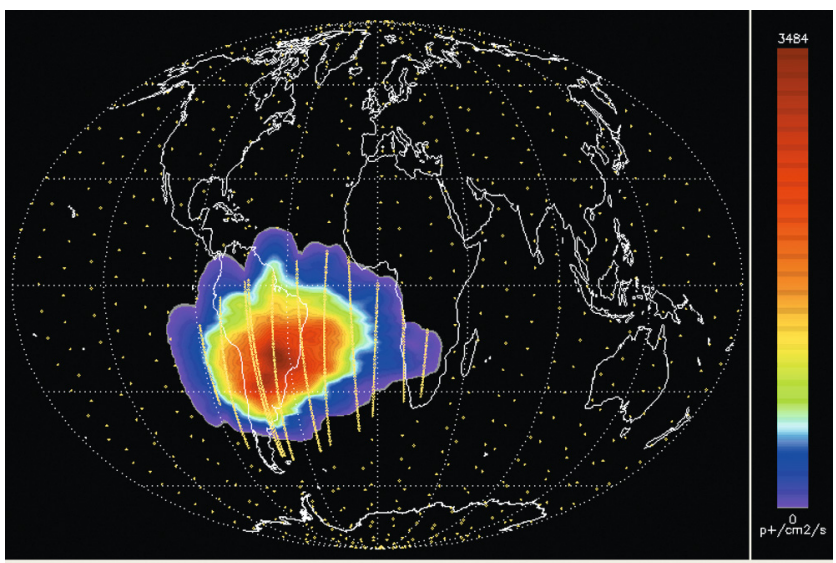

Fig. 4. Radiation flux $\mathrm{p}^{+} / \mathrm{cm}^{2} / \mathrm{s}$ map. The yellow points are the moment of image acquisition.The oscillations on the edges come from a poor time sampling.

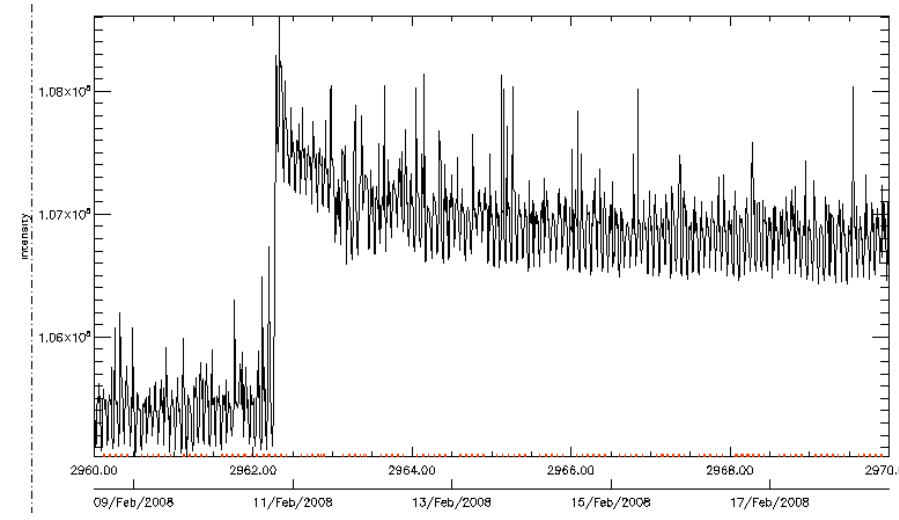

Fig. 5. Example of a background raw-data light curve (LC) perturbed by a new hot pixel appearing during the crossing of the SAA. In this example the hot pixel intensity decreases exponentially on a 1-day time scale and reaches a stable but higher mean value. Orbital straylight oscillations are also clearly seen.

specification, which was $90 \%$. The measured proton flux mapped onto the Earth shows the location of the SSA as in Fig. 4.

Along with transient events, proton impacts produce permanent hot pixels due mainly to atomic displacements in the silicon lattice (Srour et al. 2003; Hopkinson 1996). The intensity of a bright pixel is not stable in time. On short time scales (few minutes to few hours), rearrangement phase induces abrupt or an exponential decrease in the intensity. A long term annealing often follows, and a bright pixel can disappear after several days to 
Table 1. Number of bright pixels at the beginning of the four runs.

\begin{tabular}{lccc}
\hline \hline Date & $>300 \mathrm{e}^{-}$ & $>1000 \mathrm{e}^{-}$ & $>10000 \mathrm{e}^{-}$ \\
\hline Launch + 35 days & $22314(0.53 \%)$ & $4280(0.1 \%)$ & $33(0.00079 \%)$ \\
Launch + 105 days & $49026(01.17 \%)$ & $7479(0.18 \%)$ & $57(0.0014 \%)$ \\
Launch + 137 days & $69275(1.65 \%)$ & $13865(0.33 \%)$ & $92(0.0022 \%)$ \\
Launch + 294 days & $108435(2.58 \%)$ & $16734(0.4 \%)$ & $101(0.0024 \%)$ \\
Launch + 434 days & $173562(8.27 \%)$ & $43440(1.03 \%)$ & $174(0.0041 \%)$ \\
End of mission $(2.5$ years) & $8.5 \%$ & $1.08 \%$ & \\
\hline
\end{tabular}

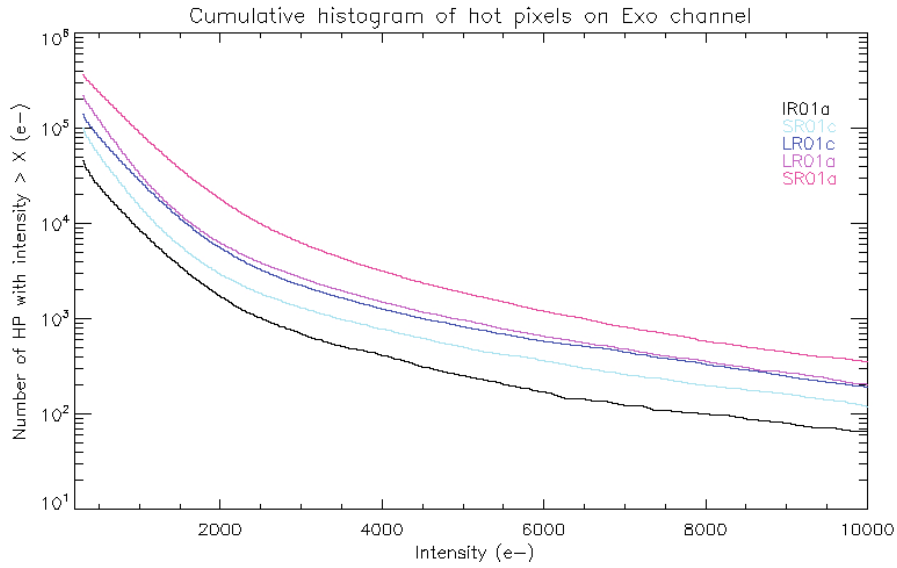

Fig. 6. Number of hot pixels having an intensity higher than $x$ electrons at the beginning of the five first runs on the PF CCDs.

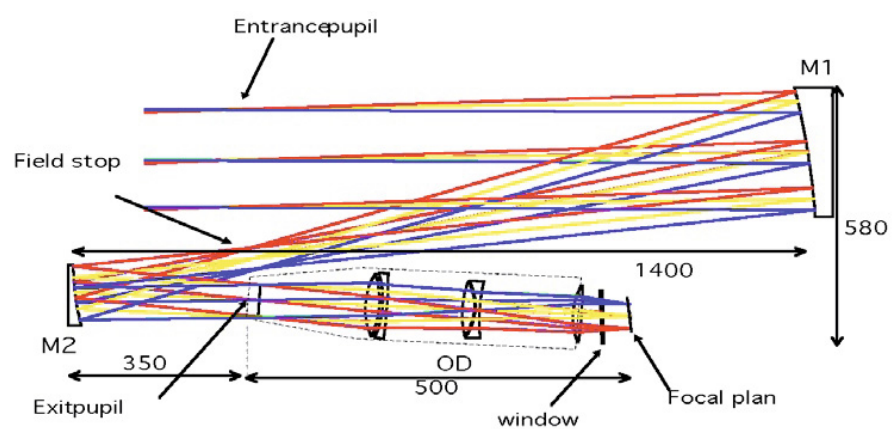

Fig. 7. Optical layout.

a few years. Figure 5 shows one example of the time behaviour of a hot pixel.

At the beginning of each run, the bright pixels are detected on PF CCDs images as a function of their intensity. The results are shown in Fig. 6 and in Table 1. The evolution of the number of hot pixels appears to be linear in time so that we can extrapolate this number to the satellite's end of life.

\section{Instrument description}

\subsection{Optics: the telescope and the focal plane}

\subsubsection{The telescope}

The optical architecture was driven by the need to minimise straylight coming from the Earth and to provide an FoV of $2.7 \times$ 3.05 degrees. This is shown in Fig. 7. An off-axis afocal telescope with a 2-stage baffle, a camera made of a dioptric objective and a focal box is the adopted solution (Viard et al. 2006). It has the following advantages: a real exit pupil and a real field stop, no central obscuration, and a cavity baffle completing the first stage. The baffling architecture is given in Fig. 8. The collecting

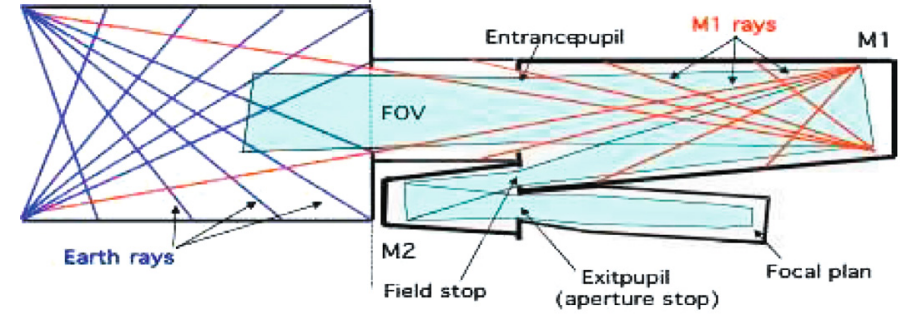

Fig. 8. Baffle architecture.

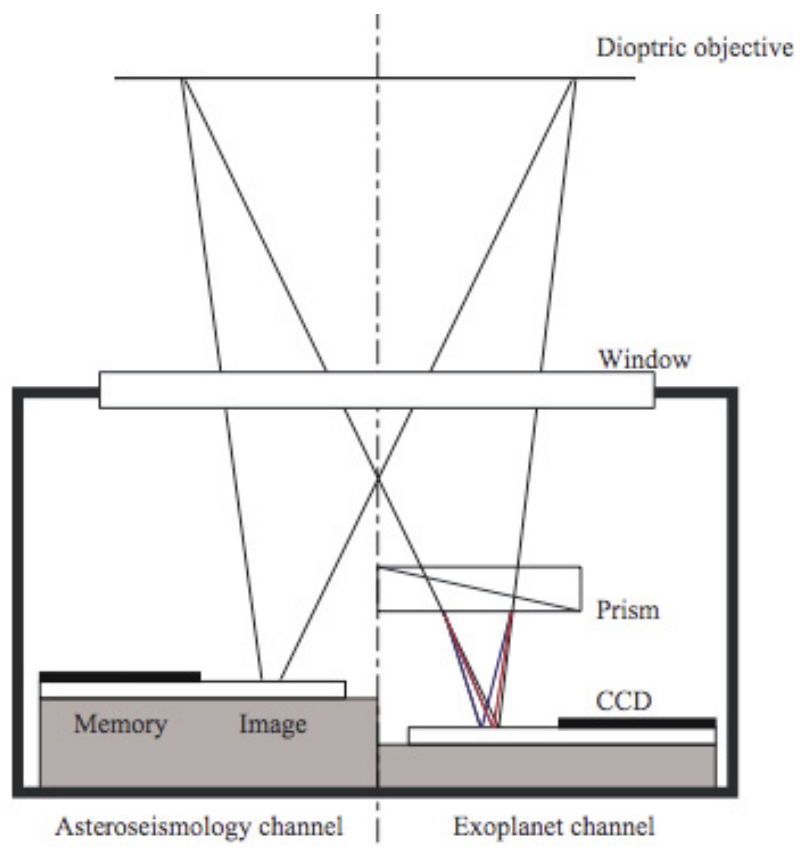

Fig. 9. Layout of the focal box.

surface is $590 \mathrm{~cm}^{2}$. With this collecting surface targets with $V$ magnitudes between 5.4 and 9.2 on the AS CCDs and with $R$ magnitudes between 11.5 and 16 on PF CCDs can be observed with a reasonable $S / N$ and without saturation of the detectors. The real pupil is at the dioptric objective entrance. To make the pupil surface variations negligible, the temperature of the pupil is stabilised. The residual orbital amplitude is less than $0.05 \mathrm{~K}$. The corresponding relative surface variation $\left(0.05 \times 10^{-6}\right)$ can be disregarded.

\subsubsection{The focal box}

The focal box contains the four CCDs. Detectors are protected against radiation by a $10 \mathrm{~mm}$ thick aluminium shielding. The AS CCDs are defocused by $760 \mu \mathrm{m}$ toward the dioptric objective to avoid saturation of the brightest stars. A prism in front of the PF CCDs gives a small spectrum. The prism dispersion 


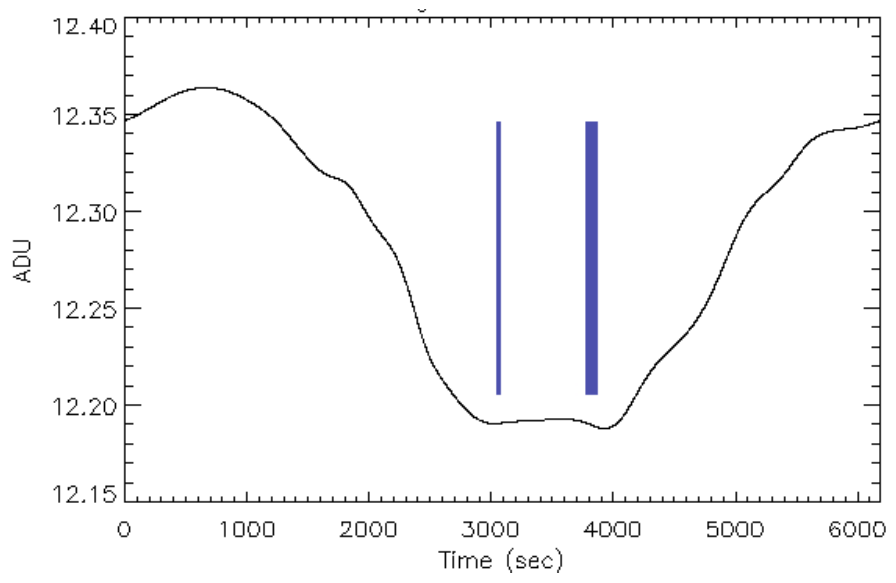

Fig. 10. Example of the background orbital variations measured on AS CCDs. Data has been folded on the orbital period. The left blue vertical bar shows the eclipse ingress and the right one the eclipse egress. The peak-to-peak amplitude is $0.2 \mathrm{ADU}$ pixel $^{-1}$ or $0.4 \mathrm{e}^{-}$pixel $^{-1}$.

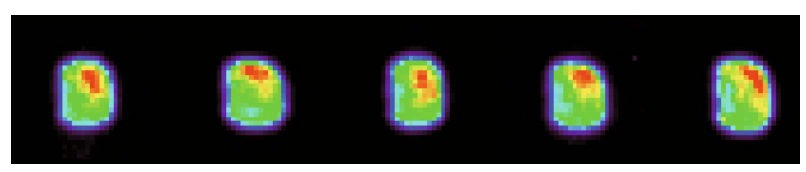

Fig. 11. Five AS PSFs. From left to right the positions on CCD A2 are: $X=1606 Y=1379, X=1901 Y=218, X=1226 Y=1907$, $X=1133 Y=795, X=72 Y=881$. The $V$ magnitudes are respectively $9.32,7.36,6.82,9.48,6.04$.

law is nonlinear, dispersing the blue more strongly than the red. The PF CCDs plane is close to the best image plane, but as the best focused image depends strongly on the wavelength, we have chosen to focus the blue to compensate for the strong blue dispersion (see Fig. 9). Evaluation of the focal plane position after the launch shows that the CCDs are defocused by $100 \mu \mathrm{m}$ more, giving a slightly larger PSF, without any consequences for the photometric precision. The focal box is thermally linked to an external radiator to keep the CCDs temperature around $-40{ }^{\circ} \mathrm{C}$ (see Sect. 4.4).

\subsection{Optical characteristics}

\subsubsection{Baffle efficiency and straylight}

The background flux is composed of several components: zodiacal light, dark current and straylight from the Earth. The straylight is responsible for the background orbital variations. Ten windows of $50 \times 50$ pixels each on the AS CCDs and 400 windows of $10 \times 10$ pixels on the PF CCDs give continuous measurements with an integration time of 1,32 , and $512 \mathrm{~s}$. The measurement precision is given by the relation

$\frac{\sigma_{B}}{B}=\sqrt{\frac{1}{k B}+\frac{\sigma_{\mathrm{r}}^{2}}{k \tau B^{2}}}$

where $B$ is the mean background per pixel per second, $\sigma_{\mathrm{r}}$ the readout noise, $\tau$ the binning window rate, and $k$ the number of pixels in the window. The binning rate is typically equal to 25 in seismology windows and 1 (no binning) in the PF background windows. With a readout noise of $8 \mathrm{e}^{-}$, an average background of $20 \mathrm{e}^{-} \mathrm{s}^{-1}$ pixel $^{-1}$, the precision is respectively $4 \%$ and $0.4 \%$ on the AS and PF CCDs. For all runs the background orbital amplitude is less than $0.6 \mathrm{e}^{-} \mathrm{s}^{-1}$ pixel $^{-1}$ (Fig. 10).

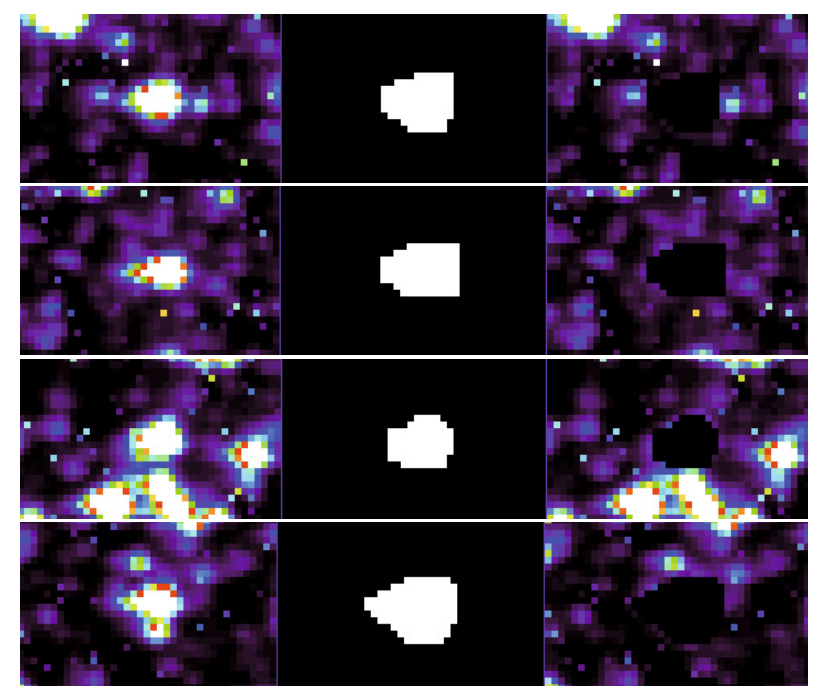

Fig. 12. Four PF PSF on CCD E1. From top to bottom the star position on CCDs is $X=1734, Y=1769, X=67, Y=1062, X=434$, $Y=317, X=40, Y=716$. The stars $R$ magnitude are respectively $12.6,13.1,13.0,13.1$. In each panel from left to right are shown the star image, the aperture and the field outside the aperture. See also Sect. 5.2.

\subsubsection{The PSF on the two channels}

Stellar images are close to simulated ones, and the diameter of AS PSF is 2 pixels larger than expected (Fig. 11); the PF CCDs plane being close to best focus the PSF is almost unchanged (Fig. 12).

\subsubsection{The geometric model}

The aim of the geometric model is to transform the coordinates of the stars into $(X, Y)$ coordinates in the CCD frames and vice versa. Four reference systems are used. (1) The equatorial J2000 inertial reference system is used to indicate the direction of stars and the orientation of the telescope. (2) The spacecraft mechanical reference system is used to describe the incident angles of the targets. (3) The focal plane reference system refers to the position of the detectors in the focal plane, and (4) the 4 CCD reference systems are used to indicate the coordinates of the stars on the detectors. The geometric model implements the transformations between these reference frames. The parameters of the geometric model are the quaternion giving the orientation of the telescope, the coefficients of distortion of the optical system, the position (in $\mathrm{mm}$ ) and orientation of the detector in the focal plane reference system, and the squared pixel size (in microns). The initial parameters of the geometric model have been derived from the optical (Zemax) model of the telescope. The parameters were updated during the commissioning run (IRa01). The method used to update the parameters is based on a chi-square minimisation between the projected coordinates of the targets in the CCD reference systems and the measured ones. In the AS channel, we iteratively calculate the telescope orientation from the positions in the AS field and the orientation of the two AS CCDs in the focal plane reference system. In the PF channel, we iteratively calculate the orientation of the two PF detectors in the focal plane reference system and optionally new values for the coefficients of distortion. At each step, the mean and maximum values of the residuals between the theoretical and measured star positions (in pixels) are calculated. Table 2 summarises the results obtained during the first run IRa01. Figure 13 shows the 
Table 2. In-flight position and orientation of the AS and PF detectors in the focal plane reference system, as derived during the commissioning run.

\begin{tabular}{llc}
\hline \hline CCD & Parameter & Value \\
\hline A1 & $X(1,1)$ - coordinate offset of the first pixel in the focal plane reference system & -14 microns \\
& $Y(1,1)-$ coordinate offset of the first pixel in the focal plane reference system & -20 microns \\
& Rotation angle in the focal plane reference system & -0.03 degrees \\
\hline A2 & $X(1,1)$ - coordinate offset of the first pixel in the focal plane reference system & -9 microns \\
& $Y(1,1)-$ coordinate offset of the first pixel in the focal plane reference system & -2 microns \\
& Rotation angle in the focal plane reference system & -0.05 degrees \\
\hline E1 & $X(1,1)$ - coordinate offset of the first pixel in the focal plane reference system & -60 microns \\
& $Y(1,1)-$ coordinate offset of the first pixel in the focal plane reference system & -11 microns \\
& Rotation angle in the focal plane reference system & -0.01 degrees \\
\hline E2 & $X(1,1)$ - coordinate offset of the first pixel in the focal plane reference system & -43 microns \\
& $Y(1,1)-$ coordinate offset of the first pixel in the focal plane reference system & +60 microns \\
& Rotation angle in the focal plane reference system & -0.01 degrees \\
\hline
\end{tabular}
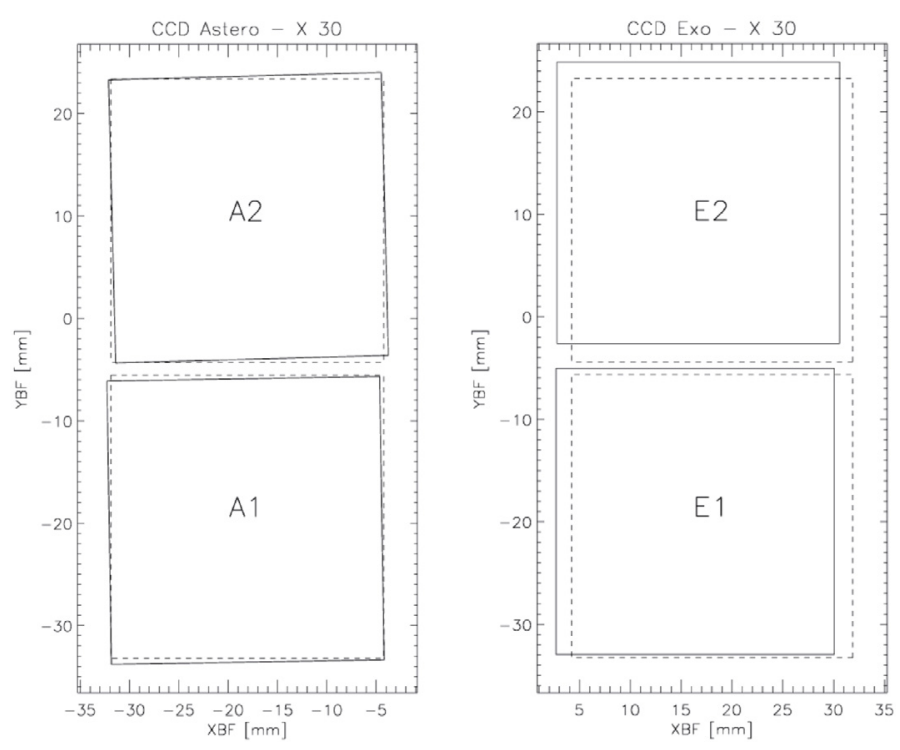

Fig. 13. Positions and orientations of the detectors in the AS and PF field. All the offsets have been amplified by a factor 30 . The dashed line squares show the location of the detectors in the optical model. The solid line shows the location of the detectors as derived after the commissioning run IRa01. XBF and YBF is the focal bloc reference frame (unit: millimeters). The CCDs origins are given in this reference frame.

position and orientation of the detectors in the asteroseismology and exoplanet fields. The maximum offset amounts to $60 \mu \mathrm{m}$ or 4.5 pixels. The residuals amount to 0.3 pixels (mean) and 0.7 pixels (maximum) for the AS field, and 0.3 pixels (mean) and 0.9 pixels (maximum) for the PF field, without updating the coefficients of distortion. Attempts to improve the coefficients of distortion in the exoplanet fields yield a decrease in the mean residuals by less than $20 \%$ and do not change the maximum residuals significantly. A new calibration made in the beginning of April 2008 show a translation of the PF field along the $Y$ CCD axis of 2.5 pixels or $33.7 \mu \mathrm{m}$.

\subsubsection{The transmission}

The transmissions of all optical subsystems were measured before launch. The transmission for the two channels is plotted in Fig. 14. We can see that the main effect of the lenses and prism is to cut the efficiency bandwidth on the blue side at $400 \mathrm{~nm}$. The prism reduces the transmission on the PF channel slightly.

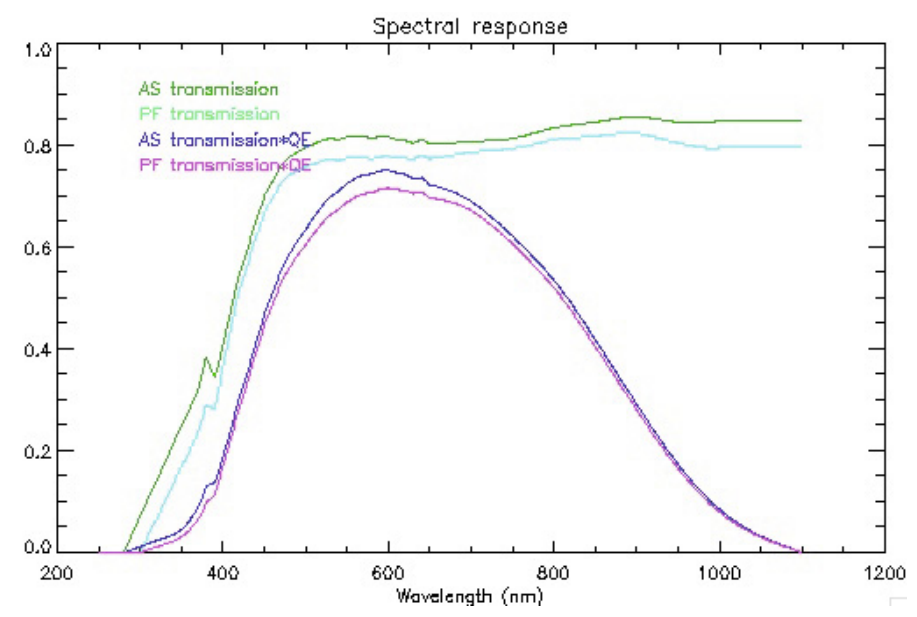

Fig. 14. Spectral response for the two channels. Differences between $\mathrm{AS}$ and PF come from to the prism absorption. The quantum efficiency (QE) has been measured for 9 wavelength values. It is the mean value for the four CCDs.

An evaluation of the global transmission in flight can be obtained by comparing measured fluxes of real stars with computed values, using model atmospheres and the transmission measured before the launch. The computation of the flux uses the Kurucz (Barban et al. 2003) atmosphere models with the effective temperature in the interval $9000-3500 \mathrm{~K}$ and a surface gravity of 4.5 as the main parameters. After identifying the targets using Tycho 2 or the Exodat catalogues (Deleuil et al. 2009), a magnitude and an effective temperature are available. For the AS programme the $V$ magnitude is computed from the Tycho catalogue with the relation $V=V_{\mathrm{T}}-0.1284\left(B_{\mathrm{T}}-V_{\mathrm{T}}\right)+0.0442\left(B_{\mathrm{T}}-V_{\mathrm{T}}\right)^{2}-$ $0.015\left(B_{\mathrm{T}}-V_{\mathrm{T}}\right)^{3}$ and the effective temperature is given from the seismology data base (Charpinet et al. 2006; Solano et al. 2005). For the PF programme, the $V$ magnitude and a colour temperature are directly found in the Exodat catalogue. The efficiency given in Fig. 14 is used for photon-to-electron conversion.

The flux is measured on full-frame images obtained at the beginning of each run. More than 120 stars are identified on each AS CCD for the three runs IRa01, LRc01, and LRa01. To minimise the measurement errors, 300 bright stars with $V$ magnitude in the interval 11.5-13.5, are processed in the same way on the PF field. Stars with temperatures hotter than $9000 \mathrm{~K}$ or cooler than $3500 \mathrm{~K}$ are eliminated and the relation "computed flux" versus "measured flux" is determined.

The slope is of the order of 0.94 with a dispersion of 0.05 , and the measured flux seems slightly larger than the computed 


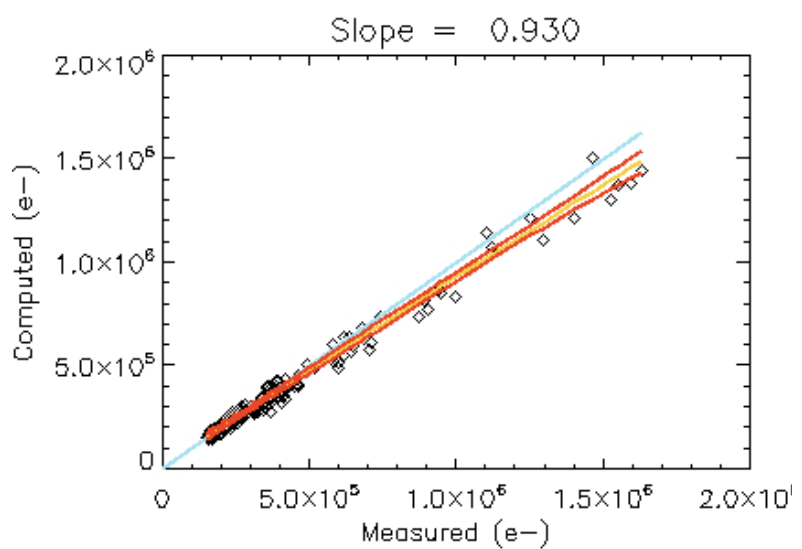

Fig. 15. Comparison of the computed and measured flux for the AS CCDs. Green curve: line of slope 1. Yellow curve: best polynomial fit. Red curves: one $\sigma$ error limits on the polynomial coefficients.

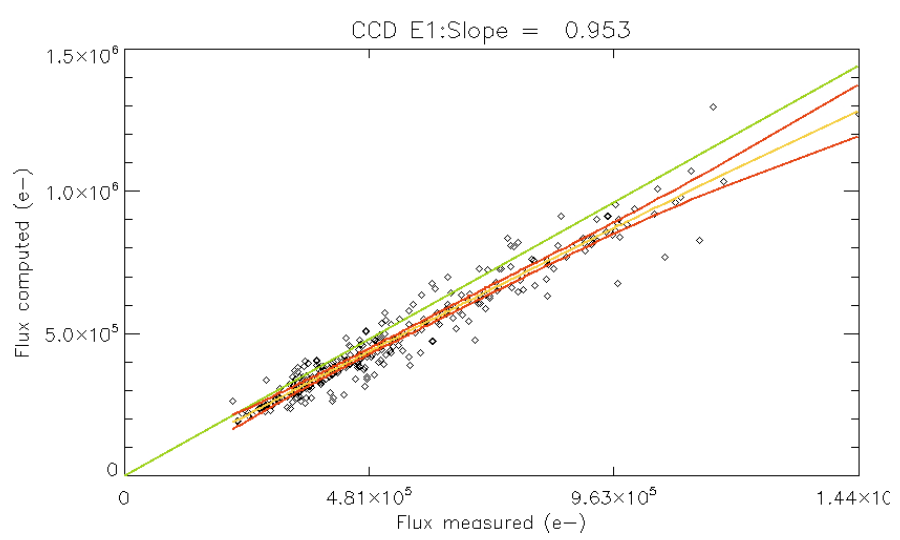

Fig. 16. Comparison of the computed and measured flux for the PF CCD E1. Green curve: line of slope 1. Yellow curve: best polynomial fit. Red curves: one $\sigma$ error limits on the polynomial coefficients.

one. This discrepancy could stem from the poor sampling of the quantum efficiency measurements (see Figs. 15 and 16).

\subsection{The $C C D$ and readout chain}

\subsubsection{The CCD detectors}

The focal plane is composed of four CCDs, namely 4280 CCDs provided by E2V Technologies. These are frame-transfer, thinned, back-illuminated detectors presenting $2048 \times 2048$ pixels in the image zone, and a memory area of $2048 \times 2054$ pixels, and each pixel is $13.5 \times 13.5 \mu \mathrm{m}^{2}$ in size. The corresponding angular pixel size is 2.32 arcsec.

The four CoRoT CCDs are denoted as A1, A2 when belonging to the AS programme, and E1 and E2 for the PF programme. Each CCD has two outputs and consequently eight electronic chains are necessary for the digitisation.

The flight detectors were selected from among 10 candidates (see Lapeyrere et al. 2006, for a description of the calibration and sorting). The quantum efficiency limits the bandwidth in the wavelength interval 250 to $1000 \mathrm{~nm}$ (see Fig. 17).

On the AS CCDs, the acquisition cycle is $1 \mathrm{~s}$., so there is no time for a whole image readout of $22.70 \mathrm{~s}$, but this is possible on the PF CCDs, with a cycle of $32 \mathrm{~s}$. In nominal observing mode only ten windows $(50 \times 50$ pixels $)$ can be extracted from each $\mathrm{CCD}$, five star windows and five background windows binned $5 \times 5$. Additionally an overscanned row of 2048 pixels allows the

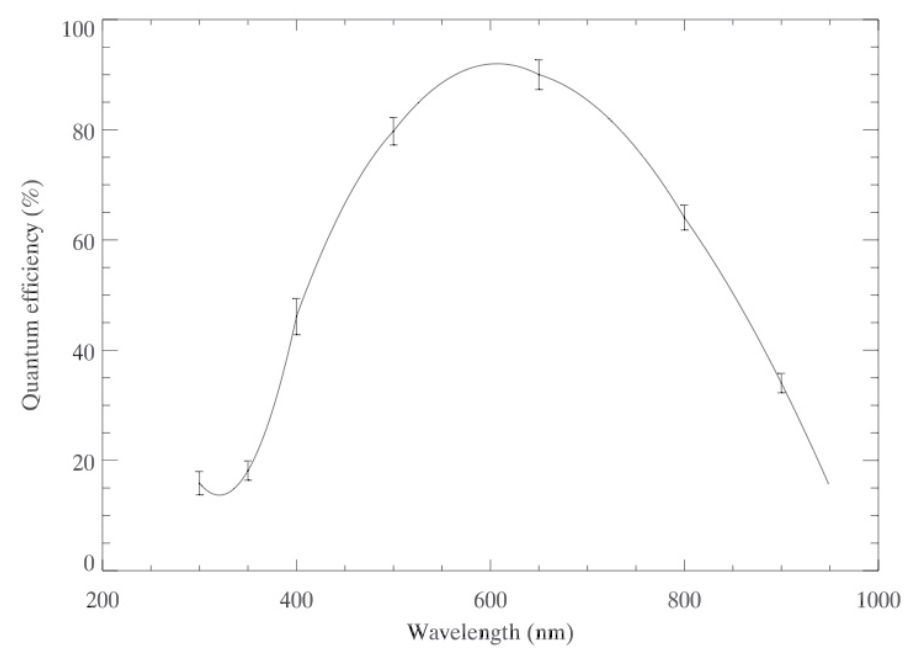

Fig. 17. Mean quantum efficiency of 4280 CCDs.

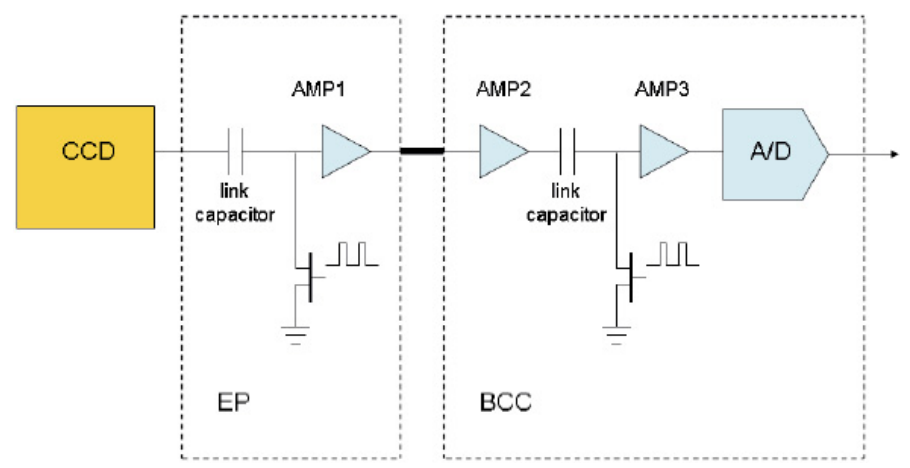

Fig. 18. Schematic of the readout electronics.

measurement of the zero level of each left and right electronic readout chain.

The exposure duration is limited by the beginning of charge transfer between the image zone to the memory zone. The row transfer on the AS CCDs lasts $100 \mu$ s and $150 \mu$ s on the PF CCDs. Consequently, the image exposure times are respectively $0.7953 \mathrm{~s}$. and $31.61928 \mathrm{~s}$.

The CCDs are operated at about $-36{ }^{\circ} \mathrm{C}$. giving a theoretical dark current rate of $0.6 \mathrm{e}^{-} / \mathrm{pixel} / \mathrm{s}$. Such a value is difficult to measure. It would require a perfectly hermetic shutter, which is not the case.

\subsubsection{The readout electronics}

The readout electronics are responsible for the digitisation of the output signal from each half CCD. A schematic view is shown in Fig. 18. A pre-amplification stage (called EP) is placed in the vicinity of the CCD. The following signal processing is performed by the control camera unit (called BCC in Fig. 18). Digitisation is performed by a 16 bit A/D converter, for an input signal ranging from 0 to $5 \mathrm{~V}$, which corresponds to $76.3 \mu \mathrm{V} / \mathrm{ADU}$. The CCD capacity being $120000 \mathrm{e}^{-}$the total gain is about $2 \mathrm{e}^{-} / \mathrm{ADU}$.

The estimate of the gain exploits the fact that if the statistical distribution of detected photons has a Poisson distribution, it is not preserved after readout when it is passed through a non unitary gain block (Janesick 2001; Pinheiro da Silva et al. 2006). We compute the relation mean variance for images of different intensity levels. The proportionality factor represents the gain 

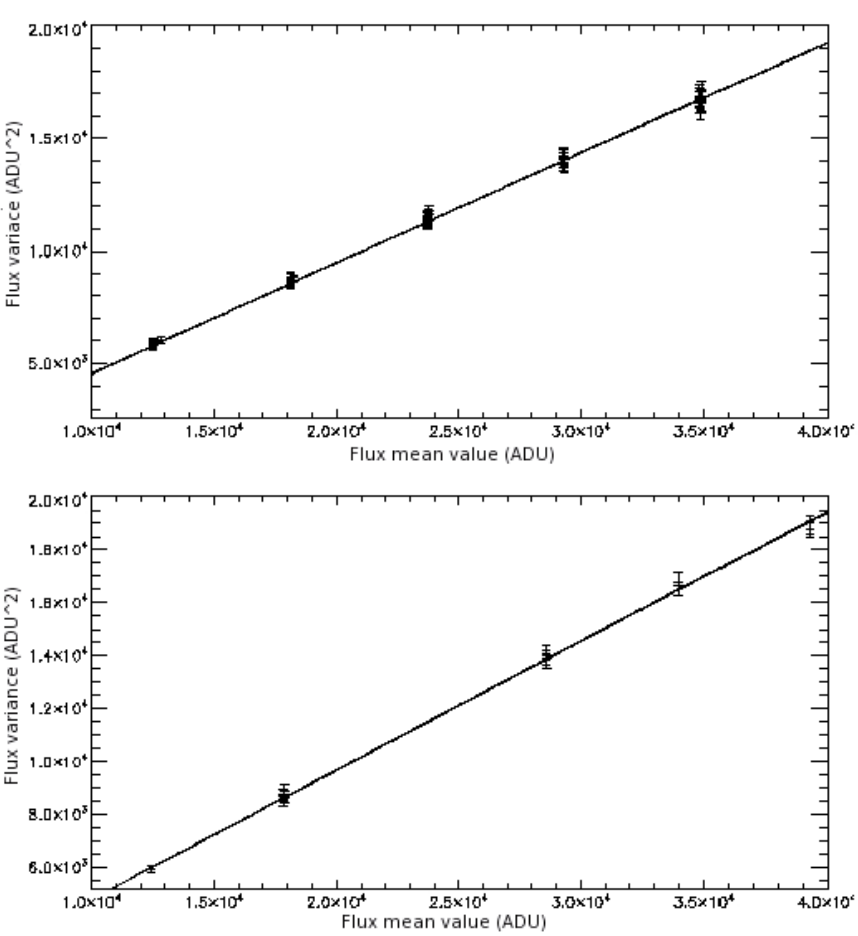

Fig. 19. Mean-Variance relation for two CCDs. Top: A1, bottom: E1.

Table 3. Readout noise and gain. where $\mathrm{L}$ and $\mathrm{R}$ stand for left and right CCDs output.

\begin{tabular}{lcccc}
\hline \hline CCD name & A1L & A1R & A2L & A2R \\
\hline $\begin{array}{l}\text { Readout noise } \\
\text { (ADU/pixel/readout) }\end{array}$ & 4.34 & 4.26 & 4.25 & 4.36 \\
\hline Gain (e $\mathrm{e}^{-} /$ADU) & 2.04 & 2.05 & 1.99 & 1.99 \\
\hline CCD name & E1L & E1R & E2L & E2R \\
\hline $\begin{array}{l}\text { Readout noise } \\
\text { (ADU/pixel/readout) }\end{array}$ & 5.5 & 5.4 & 5.5 & 5.3 \\
\hline Gain (e $\mathrm{e}^{-} /$ADU) & 2.06 & 2.05 & 2.15 & 2.14 \\
\hline
\end{tabular}

value itself. To avoid the pixel response non uniformity contribution and low frequency non-uniform contributions to the variance, it is computed on an image which is the difference of two independent images with the same mean intensity level (see Fig. 19 and Table 3 for the results). In this case the proportionality factor is twice the gain.

The readout noise is measured through the readout of a preemptied CCD output register. This line, called the offset line, is registered at each exposure on the eight half-CCDs (Table 3).

All stars show a long term decrease in the flux that is roughly linear in time. The slope, measured on the 146 days of the run LRc01 for the ten AS stars, varies linearly with the absolute flux value. Attributing the flux decrease to a gain decrease, the gain variation is close to $-1 . \times 10^{-4}$ days $^{-1}$.

\subsubsection{Cross talk perturbations}

The main cross-talk perturbations are between the PF and AS CCDs. As said, all readout processes are synchronised, which means that along a run the cross-talk perturbations always affect the same pixels and that the perturbation intensity is stable on a long time scale (>32 s). Fifteen different low-level commands are used for the charge transfer and pixel digitisation. The most important sequences are: pixel digitisation $10 \mu \mathrm{s}$, line transfer in

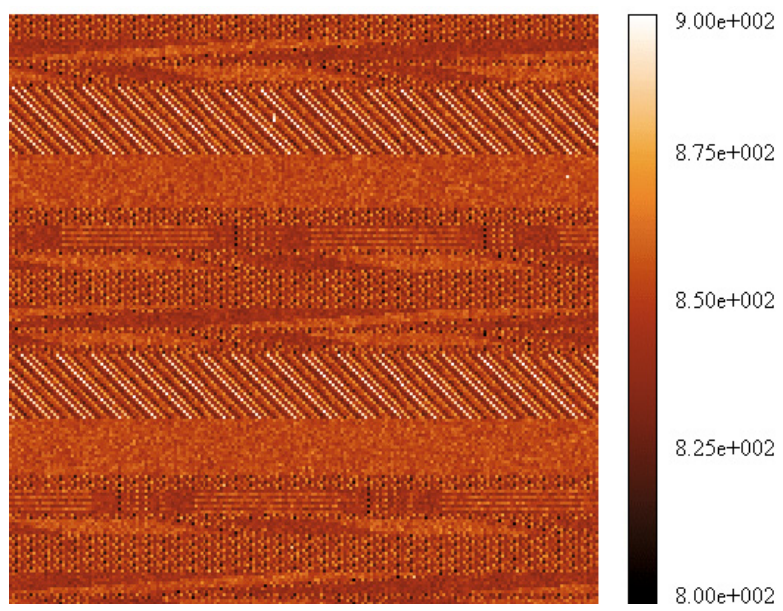

Fig. 20. Perturbation pattern on a $150 \times 150$ pixel sub-image of a PF CCD.

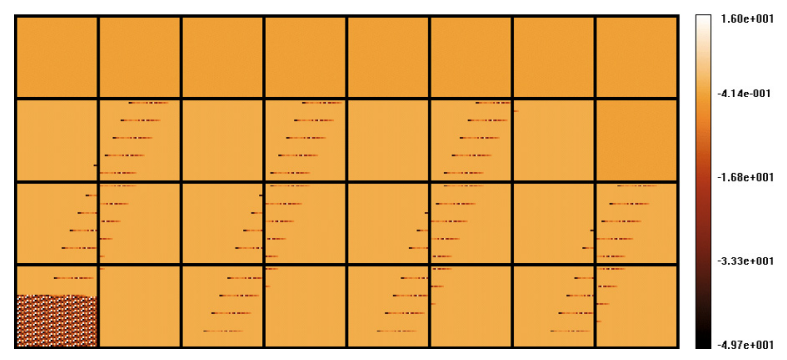

Fig. 21. Perturbation on a $50 \times 50$ pixel AS window during a 32 -s cycle. The first exposure is the bottom left image and the last exposure the upper right image. The first exposure is strongly perturbed by the end of the image zone to the memory zone charge transfer of the PF CCD. The nine last exposures are free of perturbations, with the PF readout lasting only $22.7 \mathrm{~s}$.

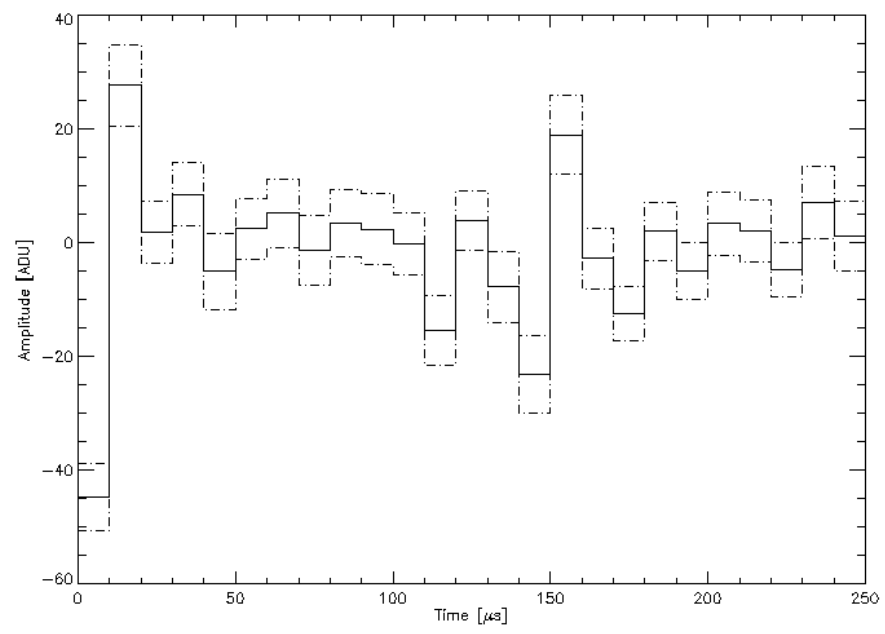

Fig. 22. Example of the perturbation on an AS CCD by the "line transfer in register" command of a PF CCD. The command lasts $250 \mu$ s and the perturbation is calibrated in $10 \mu$ s steps. The amplitude can reach 80 ADU. The dashed lines show the $1 \sigma$ uncertainty.

the register 150 and $250 \mu \mathrm{s}$, binning in the summing well $20 \mu \mathrm{s}$, clear register $100 \mu \mathrm{s}$, line transfer from the image zone to the memory zone 100 and $150 \mu \mathrm{s}$. By knowing which sequences are active at each time step, we can compute the location of the perturbation, and its intensity is calibrated for each pixel on actual images. 

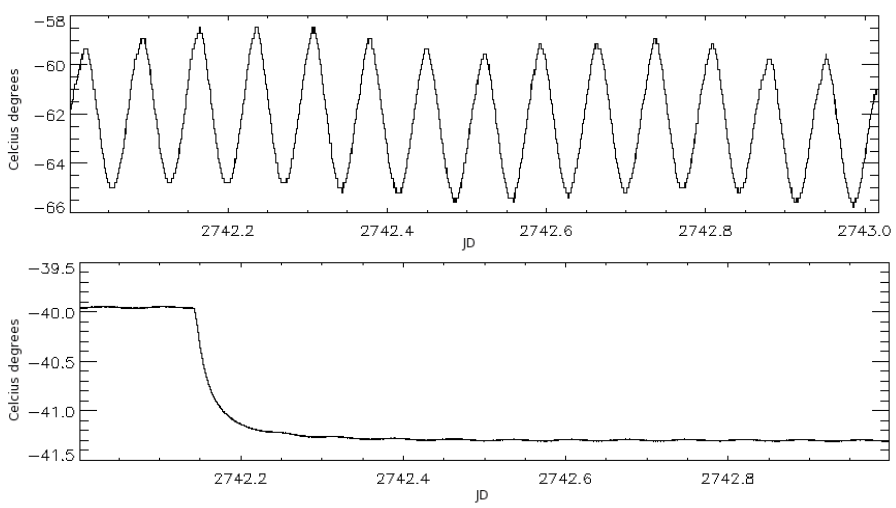

Fig. 23. Upper panel: radiator temperature during $24 \mathrm{~h}$. The orbital amplitude is 6 degrees. Lower panel: temperature of the CCDs around a target temperature jump (27th july 2007). The mean temperature jump is 1.4 degrees.

Figure 20 shows an example of cross talk on the PF CCD due to the AS readout, and Fig. 21 the perturbation during a 32-s cycle of an AS $50 \times 50$ pixel image from the PF CCD readout. Figure 22 shows the intensity of the perturbation on an AS CCD due to the PF line transfer in the register command.

\subsection{Thermal control}

To ensure the stability of the photometric signal, the main thermal requirements are:

- a telescope stability better than $0.3 \mathrm{~K}$ on the orbital time scale and lower than $1 \mathrm{~K}$ on a 150 -day run;

- a detector temperature lower than $-40{ }^{\circ} \mathrm{C}$ and a stability of $0.02{ }^{\circ} \mathrm{C}$ peak-to-peak on the orbit;

- a stability of the readout electronics better than $0.3{ }^{\circ} \mathrm{C}$ peakto-peak on the orbit.

The thermal structure is made of five isolated thermal enclosures, the external baffle, telescope, upper equipment bay including the CCD readout electronics, lower equipment bay, and the focal plane assembly. In order to reduce the orbital variation as much as possible, a systematic increase of passive thermal inertia is used by increasing the components mass. For an extensive description of the thermal design see Hustaix et al. (2007).

Ten PROTEUS thermal control heaters are used by the CoRoT payload. More specifically, there are two regulation lines in the vicinity of the mirror M2, one for the mirror M1, two lines on the dioptric objective, one on the focal plane assembly, two on the honeycomb plateau supporting the mirrors, and two on the equipment bay. A specific and very accurate heater line provides the detectors stability to smooth out the radiator's orbital and seasonal variations (Fig. 23). This heater line uses sensitive and linear sensors (AD590) and a pure proportional method using coefficients that give a quick response $(8 \mathrm{~Hz})$ of the system. As the radiator temperature varies along a run between -60 and $-80{ }^{\circ} \mathrm{C}$ the target regulation temperature must be changed several times to ensure that the CCDs orbital fluctuations are less than $0.01{ }^{\circ} \mathrm{C}$ peak to peak. Consequently the mean CCDs temperature varies from -40 to -36 degrees.

The correlation between the photometric signal and temperature variations is difficult to determine, particularly on an orbital time scale, as several subsystems can experience orbital temperature perturbations, and different physical processes may affect the LC on the same time scale. We have therefore chosen to characterise only the effect of the CCDs temperature on the LC, when
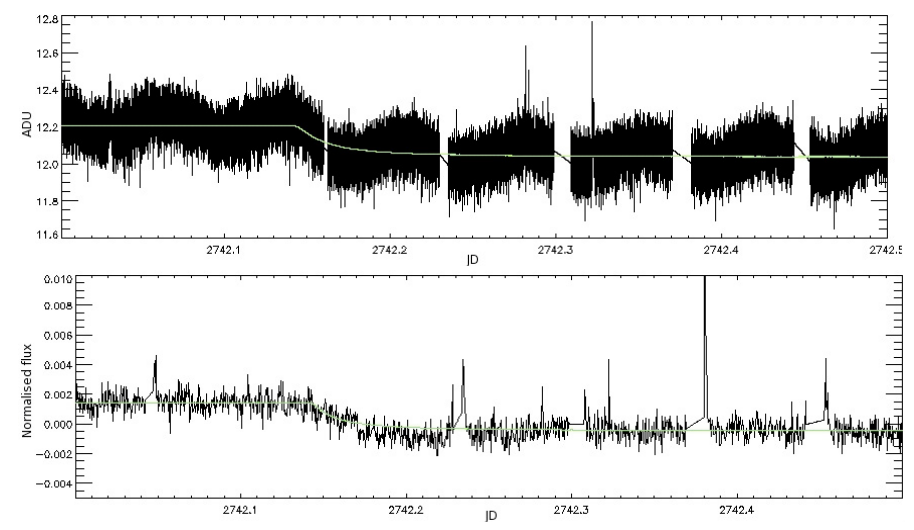

Fig. 24. From top to bottom: background LC variation on an AS CCD. Averaged and normalised PF LC for stars with $m_{\mathrm{R}}$ in the interval 13-14. The green curves are the best fit to the CCDs' temperature variations.

the mean target temperature is changed. The temperature variation looks like a "discontinuity" and is easier to characterise in the offset, background, and stars LCs.

The relation between the CCD temperature and the offset is different for the eight readout chains. For instance the offset value decreases on the AS CCDs but increases on the PF CCDs. On the AS channel, the flux variations are very small and difficult to measure. Figure 24 shows the LC decrease for a background LC on an AS CCD and averaged (see Sect. 7) and normalised PF light curves. The coefficient relating the $\mathrm{CCD}$ temperature variation to the LC is close to $0.22 \mathrm{e}^{-}$pixel $^{-1}{ }^{\circ} \mathrm{C}^{-1}$ for the background and $10 \mathrm{e}^{-}$pixel $^{-1}{ }^{\circ} \mathrm{C}^{-1}$ for a $m_{\mathrm{R}} \sim 13.5 \mathrm{star}$. We have not found a unique relation between the stars flux and the CCD temperature.

\subsection{Digital assembly and on board software}

\subsubsection{Digital assembly hardware}

The digital assembly is made of two chains managing the data from one AS CCD and one PF CCD. The two chains are identical. In each chain there are two main functions: to select the useful pixels to be processed (Extractor Box or BEX) and to process the data (Digital Processing Unit or DPU). The BEX receives all pixels digitised by the readout electronics and group pixels belonging to a star into a specific buffer. The BEX can manage different image shapes: full frame image, rectangular sub-image of various size, or pixels included in a photometric aperture.

\subsubsection{On board software}

This is divided into two parts: the primary boot software (PBS) and the APplication Software (APS) located in an EEPROM or RAM. The PBS is active when the DPU is ON and the APS starts on a telecommand (TC) sent from ground. The main PBS functions are

- initialise the DPU after power-on;

- collect and provide status telemetry data;

- receive and execute low level telecommands;

- pack telemetry in the buffer and transfer it to the PROTEUS mass memory;

- start and maintain the APS executables;

- check health and handle error events.

The APS is the set of tasks that extract the photometric signal from the images for the two channels, receives and interprets 
Table 4. Main raw data for the two programmes with time sampling.

\begin{tabular}{lccc}
\hline \hline Variable & Programme & Sampling (s) & Comment \\
\hline Mean offset & AS & 1 & Averaged on 1024 pixels, (ADU px $\left.{ }^{-1}\right)$ \\
Bright pixel number & AS & 1 & Number of pixels brighter than $n \sigma$ \\
Offset variance & AS & 1 & Readout noise, (ADU px ${ }^{-1}$ ) \\
\hline Star Intensity & AS & 1 & Total inside the aperture, (ADU) \\
Baricentre $X$ & AS & 1 & star $X$ position, (px) \\
Baricentre $Y$ & AS & 1 & position, (px) \\
\hline Background Intensity & AS & 1 & Pixels brighter than $n \sigma$ are not included in the mean, ADU px ${ }^{-1}$ \\
Bright pixel number in background window & AS & 1 & Number of pixels brighter than $n \sigma$ \\
\hline Offset & PF & 32 & mean offset, ADU px \\
Background & PF & 32 and 512 & mean background, ADU px \\
Star white flux & PF & 32 and 512 & star total intensity in the aperture, ADU \\
Star "red" flux & PF & 32 and 512 & star total intensity in the red part of the aperture, ADU \\
Star "green" flux & PF & 32 and 512 & star total intensity in the green part of the aperture, ADU \\
Star "blue" flux & PF & 32 and 512 & star total intensity in the blue part of the aperture, ADU \\
\hline
\end{tabular}

Note: on the AS channel the offset and background are subtracted on board.

the telecommands, and packs and transfers the "scientific data". The APS processes each second two offset, five background and five star windows, and every $32 \mathrm{~s}$, a maximum number of 6000 objects (5720 star, 200 background and 80 offset). The telemetry content is summarised in Table 4. The BEX, the DPU and the on board software work exactly as expected.

\subsection{On board time}

To synchronise all the readout processes a stabilised quartz of $10 \mathrm{MHz}$ inside the payload produces all signals. The basic time scales are $10 \mu \mathrm{Hz}$ for the pixel digitisation. and $100 \mu \mathrm{Hz}$ and $150 \mu \mathrm{Hz}$ for the line transfer from the image to memory zone respectively on the AS and PF CCD. A signal sent by the PROTEUS clock, named Real Time Clock (RTC), every 32 s, starts the PF CCD image to memory transfer. On the AS CCD, in a cycle of $32 \mathrm{~s}$, the first 31 transfers start on a one second signal given by the payload quartz, and the last transfer starts on the RTC signal. There is no synchronisation between the payload quartz and the RTC.

On PROTEUS a counter measures the RTC quartz frequency. Each second a GPS receiver delivers a UT date and a one second signal, the stability of which is $1 \mu \mathrm{s}$. When the counter receives this signal its value is registered and written in the housekeeping telemetry. With the UT date and the counter values we transform the on board time to UT and correct the RTC frequency fluctuations essentially due to the temperature variations of the quartz.

\subsection{The satellite attitude control system (ACS)}

The attitude control system hardware is composed of a startracker, gyroscopes, inertial wheels, and magnetotorquers. The wheel desaturation is performed by the magnetotorquers in a continuous way to avoid strong perturbations of the line of sight, which is done several time each orbit. The inertial wheels and magnetotorquer command laws are determined by a Kalman filter which receives velocity information from the star-tracker and from the gyroscopes. The stability of the standard PROTEUS ACS is 16 arcsec, far from the CoRoT specification of 0.5 arcsec. To reach the specification during the observing phases, the instrument replaces the star tracker. With two bright stars on one of the two AS CCD, the pointing error is computed each second and sent to the Kalman filter with a delay of $1.650 \mathrm{~s}$. With this improvement the rms pointing stability is $0.15 \operatorname{arcsec}$ on the

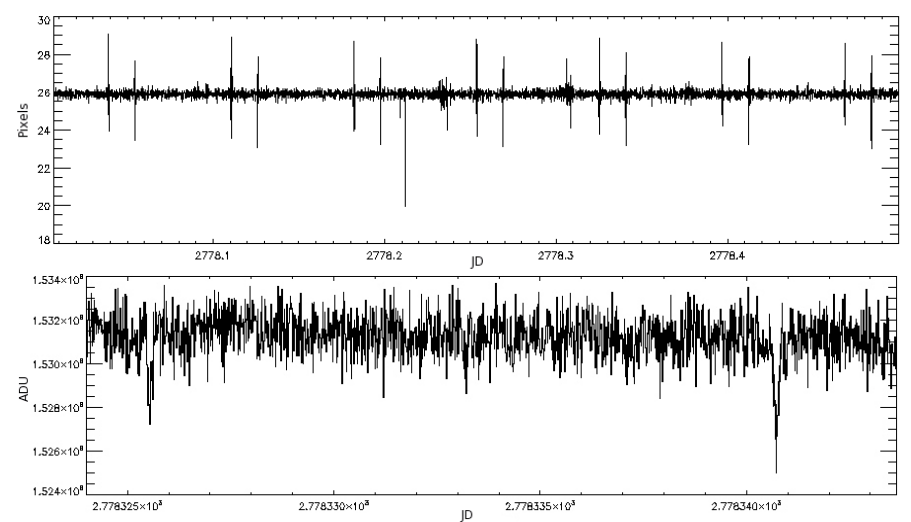

Fig. 25. Upper panel: baricentre $X$ with periodic solar array perturbations. Lower panel: detail of the light curve on an eclipse ingress and egress.

transverse axis and 5 arcsec around the line of sight. This third angle gives a negligible contribution to the field motion on the CCD. At eclipse transition (light to shadow or shadow to light), rapid temperature variations of the solar arrays (from -80 to $+70{ }^{\circ} \mathrm{C}$ ) produce vibrations and consequently attitude fluctuations twice each orbit. The amplitude can reach 12 arcsec depending of the arrays' orientation and the position of the Sun. The perturbation of the stellar light curve typically lasts $30 \mathrm{~s}$ with a delay of about $10 \mathrm{~s}$ at the light to shadow transition, and $50 \mathrm{~s}$ at the eclipse egress. An example of eclipse perturbation is given in Fig. 25 for a AS $\left(m_{\mathrm{v}}=6.74\right)$ star.

The amplitude of the pointing perturbation of the LC flux depends on the aperture, PSF shape, and the depointing amplitude. It is generally less than $0.4 \%$ but can reach $20 \%$ for eclipse ingress/egress.

The pointing error is computed with the measured position of two bright stars on one AS CCD. To obtain the best precision on the baricentre, the offset and background measured on board are first subtracted from the stellar image. Then pixels brighter than a threshold (typical value 400 ADU) are selected and used to compute the baricentre. With such an algorithm, the position measurement is sensitive to proton impacts, but this is not a real problem, because during the SAA crossing the photometric signal is also strongly perturbed independently of the pointing fluctuations. 


\section{Extracting the photometric signal}

To comply with the telemetry volume available ( $1.5 \mathrm{Gbits} / 24 \mathrm{~h})$, the photometric signal must be extracted on board for most of the targets. An aperture photometry method is used. The problem is to define the pixels belonging to the aperture giving a flux measurement that maximises a $S / N$. While it is possible to keep five arrays describing the aperture in memory in the AS channel, it is not possible for the $6000 \mathrm{PF}$ objects. The number of PF apertures limited to 255 on board, the problem is to reduce 6000 optimised apertures to 255 without a significant decrease in the $S / N$.

\subsection{Aperture on the AS channel}

As only ten stars are processed on the AS CCDs it is possible to define an aperture for each star and upload it on board. The $S / N$ is defined in a classical form as

$\frac{S}{N}(k)=\frac{\sum_{i=1}^{k} N_{\mathrm{e}}(i)}{\sqrt{\sum_{i=1}^{k}\left(N_{\mathrm{e}}(i)+\sigma_{i}^{2}\right)}}$

where $N_{\mathrm{e}}$ is the pixel intensity sorted by decreasing order. The maximum value of $S / N$ gives the set of pixels belonging to the aperture. The total noise can be defined in this simple form if its contribution is statistically independent in each pixel, but this hypothesis fails with jitter noise. All pixels on the aperture edges contribute to the jitter noise. An iterative method (Fialho \& Auvergne 2006) is used to define the aperture using as initial condition an aperture deduced from Eq. (4). The algorithm steps are:

- a super-resolved PSF is estimared;

- target and parasite star are separated by PSF fitting. If the parasite shares pixels with the target those pixels are included in the noise contribution of the $S / N$;

- the $S / N$ is computed with Eq. (4). An aperture is determined with its maximum value;

- with this aperture the photometric signal is computed on a sample of images specially downloaded for this task. Then the photometric signal is corrected with the method described in section 6 and a $S / N$ is computed;

- an edge pixel is added to the aperture and a new $S / N$ computed from the fourth step;

- the process stops when the maximum $S / N$ is reached.

\subsection{Aperture on the PF channel}

A detailed description of the method can be found in Llebaria et al. (2002) and in Llebaria et al. (2004) and an evaluation of the $S / N$ obtained in Llebaria et al. (2003).

The star fields to be observed are sometimes quite crowded and the aperture shape will depend on: 1) the PSF distribution, 2) the local background and 3) the neighbouring stars. The best aperture for each star should minimise such effects to approach the ideal $S / N$ of photon limited noise. The signal is defined by the total flux of the star included in the aperture. The noise depends mainly on

- photon noise from the star itself;

- photon noise from the background and the overlapping stars;

- residual jitter;

- focal length orbital variations (negligible);

- periodic straylight variations.
An initial aperture is optimised for each star assuming zero jitter noise. In the second step taking jitter into account, apertures are modified to give minimal variance relative to the stellar flux. The third step is to reduce the full set of apertures from all selected stars to the limited set of 256 shapes. In the last step an aperture is assigned to each star. In the worst case the final $S / N$ is reduced by $20 \%$ compared to a $S / N$ limited only by the photon noise.

Four examples of aperture assignment can be seen in Fig. 12. From top to bottom, the two first panels show the aperture of an isolated star. The third one shows that the aperture is smaller than for the second case, and placed in such a way that parasite stars just below are not included in the aperture. The fourth panel shows a parasite star inside the target star. In that case the aperture is large and includes the parasite.

\section{Outline of data correction}

The main processing and corrections on the raw photometric signal are roughly the same for the two programmes (Samadi et al. 2006).

- Correction of the offset and background signal from the cross-talk effect.

- Subtraction of the offset and background to the star light curve.

- Stellar flux correction of the cross-talk effect (AS only).

- Stellar flux unit transform from ADU to electrons.

- Correction of the exposure time variations.

- Correction of the jitter effect.

- Correction of the outliers.

\subsection{Background corrections on the PF channel}

The background is measured on 400 windows of $10 \times 10$ pixels in the PF field, 300 with a sampling of $512 \mathrm{~s}$ and 100 with 32 s sampling; they are uniformly spread over the field in regions free of stars. The issue is how to estimate the background inside a star aperture. Four methods have been tested (Drummond et al. 2008) they are: closest background, triangular interpolation, two dimensional polynomial fit and the median of all background windows. The last two methods are the most efficient and are insensitive to bright pixels but the median is overall the most effective to correct orbital background variations, despite the fact that all the stars LCs on one CCD are corrected with the same background. The bright pixel problem in background windows has been solved recently with a new version of the on board software uploaded in April 2008, the brightest pixels inside a background window image are removed before the computation of the mean.

\subsection{Jitter-effect corrections}

The jitter corrections are completed in five steps (for a detailed description of the method see Fialho et al. 2007; and Drummond et al. 2006),

- compute a high resolution PSF for all stars (1/4 of the pixels for AS channel and $1 / 2$ the pixels for PF);

- compute a least square solution of the line of sight fluctuations with stars on the AS CCDs brighter than $V=8$;

- compute the motion of all stars in the CCD reference frame from the angles obtained in the second step;

- move the high resolution PSF inside the aperture onto a uniform grid, the flux (normalised to unity) is computed for each 

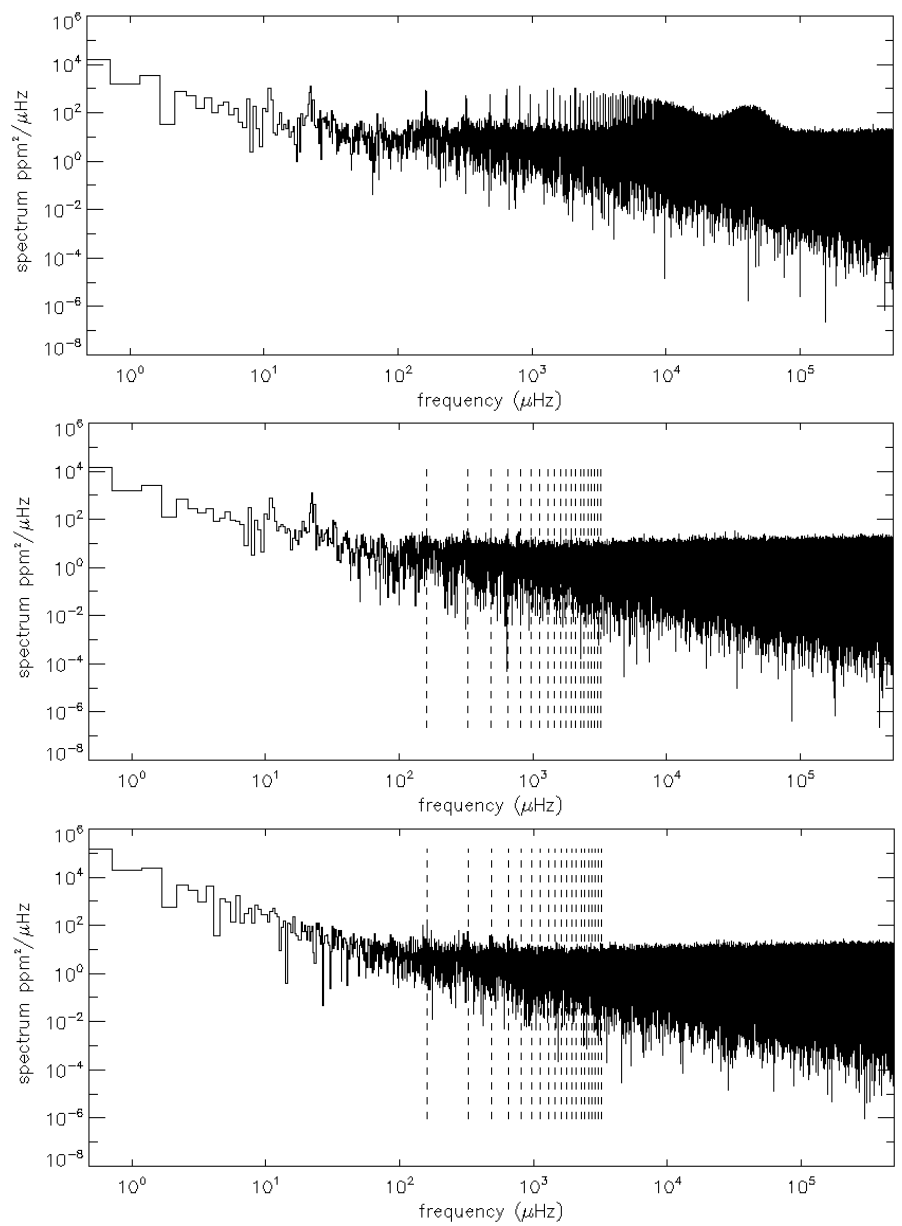

Fig. 26. From top to bottom: 1) power spectrum of a constant faint $(V=9.14)$ star in the AS channel for 15 days of data (August 2007) with eclipses, but before jitter correction; 2) spectrum of the same data after correction; 3) spectrum of the same star for 15 days data (July 2007) without eclipses and before jitter corrections. Dashed vertical lines indicate the orbital frequency and its harmonics.

grid point. This surface, computed for each target, gives the proportion of flux lost when the image moves outside the aperture;

- interpolate the surface generated in the fourth step for actual motions and compute a correction factor applied to the measured flux.

Figure 26 shows three spectra of a faint star on the AS channel. The upper one is the spectrum obtained before the jitter correction. Several lines, harmonics of the orbital frequency appear with a continuous component. This continuous component is centred at a frequency around $0.03 \mathrm{~Hz}$ (or $35 \mathrm{~s}$ ). After correction most of the lines and the continuous component disappear. The third spectrum is computed with data obtained in July 2007, a period without eclipses, and before jitter corrections. Comparing the spectra of August and July we can see that most of the lines come from eclipse ingress/egress and that a small residual remains. The efficiency of the correction depends strongly on the high resolution PSF estimation quality. Generally the PSF used to compute the correction surface is precise enough, particularly for bright stars, but sometimes the PSF is not precise enough in the PSF wings for the faintest stars. The example shown in Fig. 26 is that of a worst case.

This algorithm has not been implemented yet on the PF light curves, because the PSF computation is more difficult than for
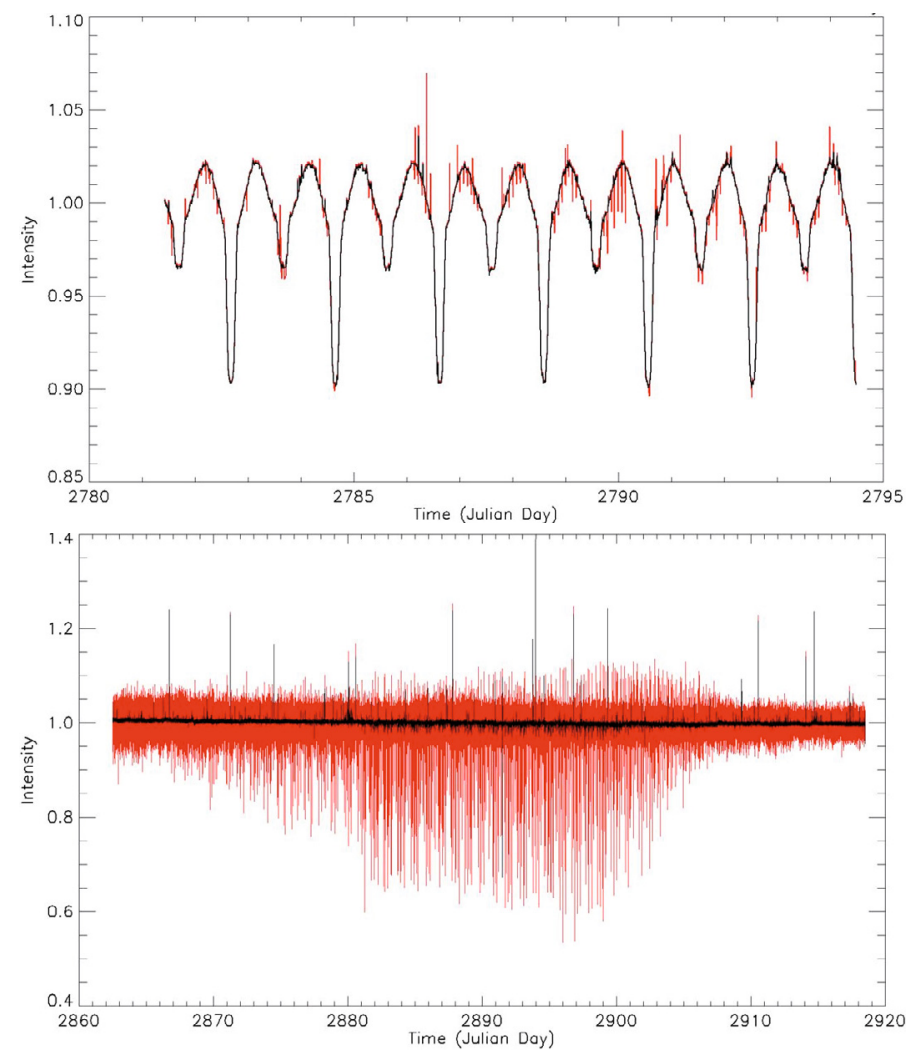

Fig. 27. Red light curve on the PF channel for two stars. Upper panel: a binary star sampled at $512 \mathrm{~s}\left(m_{\mathrm{R}}=13.7\right)$. Lower panel: a constant star sampled at $32 \mathrm{~s}\left(m_{\mathrm{R}}=14\right)$. Red curves: raw data before correction. Black curves: the signal after correction. The flux is normalised.

the AS objects, in part because the integration time is longer but also because the objects are faint and their images undersampled. But some tests have been made of the proposed method and examples of the results are shown in Fig. 27 for two stars, a binary star and a constant one. On this second star we can see a small correction residual but the perturbation is reduced by more than a factor 40 (Fialho, private comm.).

\subsubsection{Outliers}

Outliers are data points in a star's LC that are significantly larger than the average flux. Sometimes the reason for the perturbation is known or suspected (image of debris, high-energy particles) but this is not often the case. Nevertheless, outliers are removed assuming that very short photon excess cannot come from stellar flux fluctuations. The outlier detection is based on a robust and local standard deviation. Flux values that differ by more than $n \sigma$ from the mean are removed and replaced by an interpolated value.

\section{Photometric performances}

The measurement of the LC rms is carried out in Fourier space on frequencies adapted to each scientific programme. For the AS programme the noise level is measured in the frequency interval 1 to $10 \mathrm{mHz}$, which corresponds to the range of solar-like oscillations. In the PF channel the frequency interval is $2.5 \times 10^{-5}$ to $1.5 \times 10^{-4} \mathrm{~Hz}$, which corresponds to a typical transit duration. For the relation between the spectrum mean value and the rms, see for instance Bracewell (1986). 


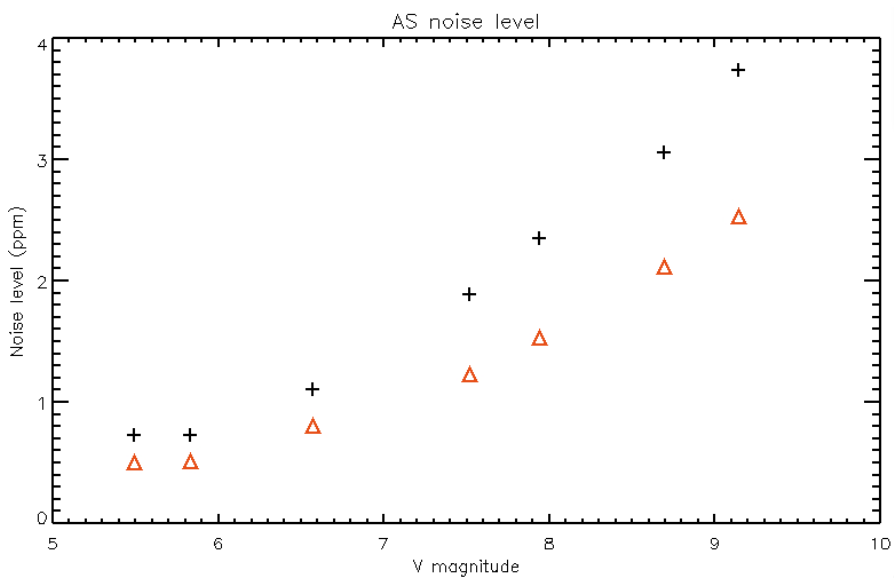

Fig. 28. Crosses: measured rms. Red triangles: photons noise level.

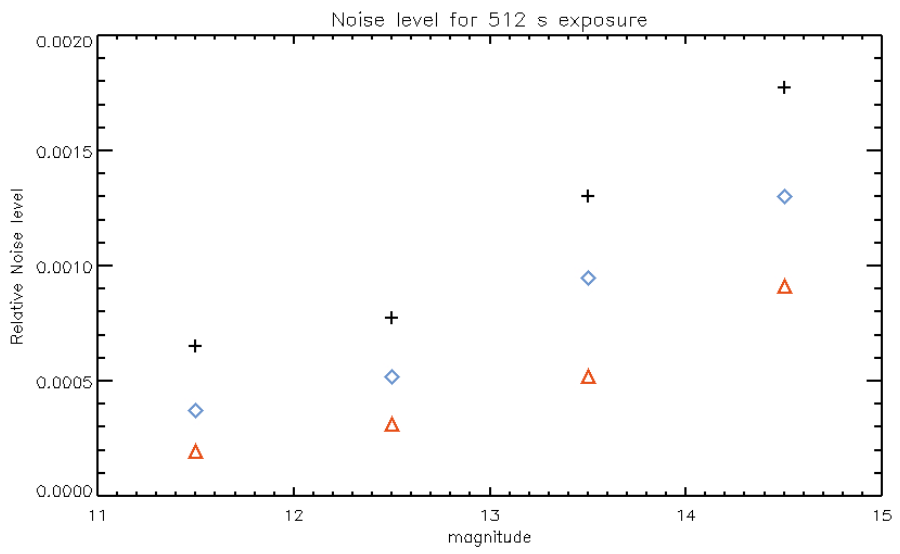

Fig. 29. Crosses: measured rms on white light curve. Blue diamond: the same but measured on a time interval without eclipses. Red triangles: white noise level (photons noise plus readout noise plus background photon noise).

\subsection{Photometric noise at high frequencies on AS channel}

The spectrum is computed using 145 days of run LRc01. The data are corrected as described in Sect. 6 and the results shown in Fig. 28. For comparison with the specification of $0.6 \mathrm{ppm}$ in 5 days (for a $V=5.7$ star) the result is normalised to correspond to a 5-day run. The equivalent photon noise is given for comparison. The small differences between the photon noise and the real one, around $V=5.8$, come from the jitter correction residual. A recent paper on the star HD 49933 (Appourchaux et al. 2008) reports the detection of several tens of solar-like p-mode oscillations with a $S / N$ down to 1.5 and amplitude less than $0.6 \mathrm{ppm} / \sqrt{\mu \mathrm{Hz}}$.

\subsection{Photometric noise at intermediate frequencies on the PF channel}

Before computing the Fourier transform, a "mean LC" is computed in four magnitude intervals. The operations performed are: select stars in a magnitude interval, normalise the mean flux to 1 and eliminate variable stars, perform a singular value analysis and reconstruct the mean $\mathrm{LC}$ on the subspace corresponding to the highest eigenvalue. The result is given in Fig. 29.

However the best performance evaluation is given by transit detection. Figure 30 shows all the transits detected in the first three runs (Barge, private comm.). More than 400 events are

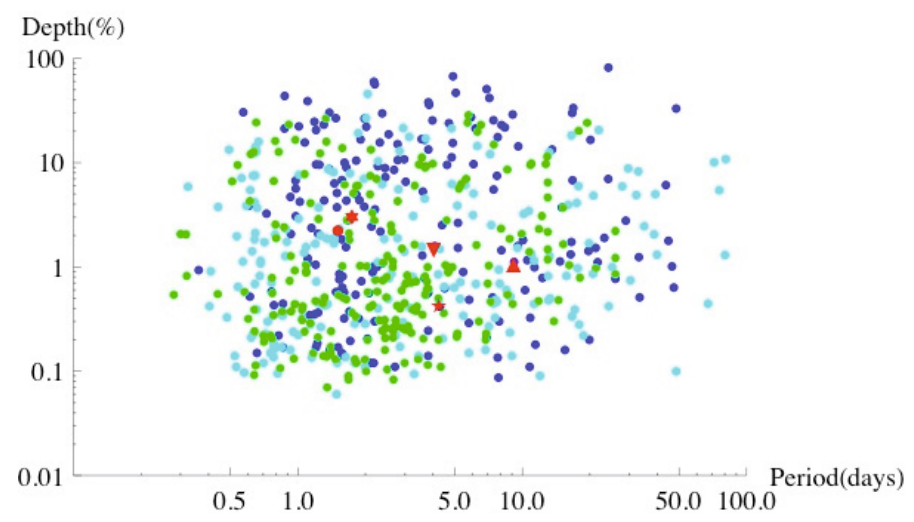

Fig. 30. Transits detected in CoRoT data during the three first runs (dark blue, light blue, and green points). Confirmed planets are red points.

reported. All the plane accessible to CoRoT is filled. The smallest transit depth is around $6 \times 10^{-4}$ (found in a star of magnitude $R=12$ ), and is compatible with the measurements shown in Fig. 29. For short or intermediate periods a large number of transits allows a detection close to the theoretical limit. The longest periods are around 75 days, which is the longest period for which we can have three transits, as required by the detection algorithms.

\section{Conclusions}

We have analysed the data of the commissioning and of four following runs. Most of the subsystems have nominal performances:

- The scattered light from the Earth is reduced by a factor about $10^{12}$ by the baffle, since the residual background orbital variations are always smaller than $0.6 \mathrm{e}^{-} \mathrm{s}^{-1}$ pixel $^{-1}$. The specification asks for a variation less than $5 \mathrm{e}^{-} \mathrm{s}^{-1}$ pixel $^{-1}$.

- The PSF is close to the expected one, despite a slightly larger defocus. The photon detection efficiency agree with calibration and transmission measurements.

- The temperature stability on short time scales is always better than the specified performances. On the CCDs the stability specification for the orbital variations was 0.03 degree, and the actual stability is better than 0.01 .

- The ACS gives a stability of the line of sight of 0.15 arcsecond, much better than the specification of 0.5 arcsec. While we did not foresee the effect of eclipse ingress/egress on the ACS, this perturbation can be efficiently corrected.

We have shown that most of the photometric perturbations come from the environmental perturbations and especially from eclipse ingress/egress. Hot pixels generated by proton impact cannot be easily corrected in stellar light curves, particularly for variable stars, but hot pixels brighter than $1000 \mathrm{e}^{-}$are rare enough $(\sim 1 \%)$ to be acceptable. The last puzzling perturbations are the temperature fluctuations that introduce variations on 24-h and orbital time scales. It is not yet clear whether we will be able to obtain a unique calibration for all stars.

\section{References}

Appourchaux, T., Michel, E., Auvergne, M., et al. 2008, A\&A, 488, 705 Barban, C., Goupil, M. J., Van't Veer-Menneret, C., et al. 2003, A\&A, 405, 1095 
Bracewell, R. N. 1986, The Fourier transform and its applications (McGraw-Hill edt.), 356

Boisnard, L., \& Auvergne, M. 2004, CoRoT mission engineering, IAC-04-IAFQ.1.01

Charpinet, S., Cuvilo, J., Platzer, J., et al. 2006, ESA SP-1306, 353

The CoRoT team, in The CoRoT Mission, Pre-Launch Status, Stellar Seismology and Planet Finding, M. Fridlund, A. Baglin, J. Lochard, \& L. Conroy, ESA SP, 1306

Deleuil, M., Meunier, J. C., Moutou, C., et al. 2009, AJ, 138, 649

Drummond, R., Vandenbussche, B., Aerts, C., Fialho, F., \& Auvergne, M. 2006, PASP, 118, 874D

Drummond, R., Lapeyrere, V., Auvergne, M., et al. 2008, A\&A, 497, 1209

Fialho, F., \& Auvergne, M. 2006, in The CoRoT Mission, Pre-Launch Status, Stellar Seismology and Planet Finding, ed. M. Fridlund, A. Baglin, J. Lochard, \& L. Conroy, ESA SP-1306, 299

Fialho, F., Lapeyrere, V., Auvergne, M., et al. 2007, PASP, 119, 337D

Hopkinson, G. R. 1996, IEEE Trans. Nuc. Science, 43, 614

Hustaix, H., Briet, R., \& Carponsin, M. 2007, International Conference on Environmental Systems, 336

Janesick, J. R. 2001, Scientific Charge-couple devices, SPIE, PM, 83

Landiech, P., \& Douillet, F. 2004, Small Satellite System and Services symposium, ed. B. Warmbein, ESA SP, 571, 1.1

Llebaria, A., Vuillemin, A., Guterman, P., \& Barge, P. 2002, Proc. SPIE, 4849, 112

Llebaria, A., Guterman, P., \& Ollivier, M. 2003, Proc SPIE, 5170, 155

Llebaria, A., Auvergne, M., \& Perruchot, S. 2004, Proc SPIE, 5249, 175

Pinheiro da Silva, L., Lapeyrere, V., \& Bernardi, P. 2006, in The CoRoT Mission, Pre-Launch Status, Stellar Seismology and Planet Finding, ed. M. Fridlund, A. Baglin, J. Lochard, \& L. Conroy, ESA SP, 1306, 309

Pinheiro da Silva, L., Rolland, G., Lapeyrere, V., \& Auvergne, M. 2006, MNRAS, 384, 1337

Pinheiro da Silva, L., Auvergne, M., Toublanc, D., et al. 2006, A\&A, 452, 363

Samadi, R., Fialho, F., Costa, J.E.S., et al. 2006, ESA SP, 1306, 317, corrected in [arXiv:astro-ph/0703354]

Solano, E., Catala, C., Garrido, R., et al. 2005, AJ, 129, 547
Srour, J. R., Marshall, C. J., \& Marshall, P. W. 2003, IEEE Trans. Nuclear Sciences, 50, 653

Viard, T., Mathieu, J -C., Fer, Y, et al. 2006, in The CoRoT Mission, Pre-Launch Status, Stellar Seismology and Planet Finding, ed. M. Fridlund, A. Baglin, J. Lochard, \& L. Conroy, ESA SP-1306, 187

1 Observatoire de Paris, UMR 8109, 5 place J. Janssen, 92195 Meudon, France

2 Centre National d'Études Spatiales, 18 avenue E. Belin, Toulouse, France

3 Laboratoire d'Astrophysique de Marseille, Traverse du Siphon, 13376 Marseille Cedex 12, France

4 Institute of Planetary Research, DLR, Rutherfordstr. 2, 12489 Berlin, Germany

5 Institut d'Astrophysique Spatiale, Université Paris XI, 91405 Orsay, France

6 DLR, German Aerospace Center, Institute for Robotics and Mechatronics, Department Optical Information Systems, Rutherford Strasse 2, 12489 Berlin-Adlershof, Germany

7 Technologiepark 1, 15236 Frankfurt/Oder, Germany

8 CLIPhIT, Arnfriedstrasse 17, 12683 Berlin, Germany

9 European Space Agency, ESTEC, SCI-A, PO Box 299, 2200AG, Noordwijk, The Netherlands

10 Space Research Institute, Austrian Academy of Science, Schmiedlstrasse 6, 8042 Graz, Austria

11 Centre Spatial de Liége, ULG Science Park, Av. du Pré-Aly 4031 Angleur-Liége, Belgium

12 Observatoire Midi-Pyrénées, 14 Av. E. Belin, Toulouse, France

13 Instituut voor Sterrenkunde, Departement Natuurkunde en Sterrenkunde, Katholieke Universiteit Leuven, Celestijnenlaan 200 D, Office 03.31, 3001 Leuven, Belgium 